Authors: Baptiste Coxam 1,2, Yvonne Padberg 3 Katja Maier 1, Simone Jung 1,

Eireen Bartels-Klein 1, Anna Szymborska 1, Lise Finotto 5, 6, Christian S.M. Helker 7, 8, Didier Y.R. Stainier 7, Stefan Schulte-Merker 3, Holger Gerhardt 1,2,4.

\title{
Svep1 stabilizes developmental vascular anastomosis in reduced flow conditions.
}

\author{
Affiliations: \\ 1 - Integrative Vascular Biology Lab, Max-Delbrück Center for Molecular Medicine in the Helmholtz Association (MDC), \\ Robert-Rössle-Strasse 10, Berlin 13125, Germany \\ 2 - DZHK (German Center for Cardiovascular Research), partner site Berlin \\ 3-Institute of Cardiovascular Organogenesis and Regeneration, Faculty of Medicine, WWU Münster, Mendelstraße 7, 48149 \\ Münster, Germany \\ 4 - Berlin Institute of Health (BIH), Berlin, Germany \\ 5 - Vascular Patterning Laboratory, Center for Cancer Biology, VIB, Leuven, Belgium \\ 6 - Vascular Patterning Laboratory, Department of Oncology, KU Leuven, Leuven, Belgium \\ 7 - Department of Developmental Genetics, Max Planck Institute for Heart and Lung Research, Bad Nauheim, \\ 61231, Germany \\ 8 - Philipps-University Marburg, Faculty of Biology, Cell Signaling and Dynamics, Marburg, 35043, Germany
}

\section{corresponding author:}

\section{Prof. Dr. Holger Gerhardt}

Integrative Vascular Biology

$\mathrm{BIH}$ and DZHK professorship for Experimental Cardiovascular Research at the Charité Robert-Rössle-Straße 10

13125 Berlin, Germany

Phone: +49 (0)30 9406-1780

Fax: $+49(0) 309406-1771$

holger.gerhardt@mdc-berlin.de 


\section{Abstract}

We report the discovery that flow and Svep1 are modulator of vessel anastomosis during developmental angiogenesis in zebrafish embryos. We show that loss of Svep1 and blood flow reduction both contribute to defective anastomosis of intersegmental vessels. We show that this defect in primary angiogenic sprouts is associated with an expansion of Apelin-positive tip cells and with reduced formation and lumenisation of the dorsal longitudinal anastomotic vessel. Mechanistically, our results suggest that flow and Svep1 act synergistically to modulate vascular network formation in the zebrafish trunk.

\section{Non-standards Abbreviations and Acronyms}

DLAV: dorsal longitudinal anastomotic vessel

hpf: hours post-fertilization

ISVs: intersegmental vessels

DA: Dorsal Aorta

PCV: Posterior Cardinal Vein

VEGFR-2: vascular endothelial growth factor receptor-2

Kdr; Kinase insert domain receptor

Kdrl: Kinase insert domain receptor like

VEGFA: vascular endothelial growth factor A

MO: Morpholino 
Angiogenesis defines the formation of new vessels from pre-existing ones and is a stepwise process that leads to the formation of a perfused network of arteries and veins that are optimally organised to serve the metabolic needs of the developing embryo. In zebrafish embryos, vasculogenesis results in the formation of the two main blood vessels, namely the dorsal aorta (DA) and the posterior cardinal vein (PCV). Later, angiogenic sprouts emerge from the DA. These multicellular sprouts are composed of a leading tip cell followed by stalk cells [1], and migrate dorsally in between the vertical somite boundaries to form intersegmental vessels (ISVs)[2]. The proliferation and migration of ISVs to the dorsal part of the embryo is driven by Vegfa signalling, through binding to the VEGFR2 zebrafish ortholog Kdr and its ohnolog Kdrl [3-8]. Activation of Kdrl signalling in the leading ISV endothelial cell (tip), mediated in large part via downstream phosphorylation of the serine/threonine kinase ERK1/2, promotes migratory behaviour. In parallel, activation of Kdrl signalling in the tip cell leads to Notch mediated inhibition of $\mathrm{Kdrl}$ signalling in trailing cells (stalks), preventing their conversion into tip cells and promoting their proliferation to support ISV expansion [6, $9,10]$. Around 30-32 hours post-fertilisation (hpf), the leading tip cells of the ISVs start to anastomose with their ipsilateral neighbours, a process that ultimately leads to the formation of the dorsal longitudinal anastomotic vessel (DLAV), dorsal to the neural tube $[11,12]$. The DLAV is initially a paired bilateral structure that is fully lumenised by $48 \mathrm{hpf}$, but subsequently both sides progressively connect to form a complex plexus $[2,12]$. Zygmunt and colleagues demonstrated that maturation of the DLAV plexus is regulated by flow and Vegfr signalling after $48 \mathrm{hpf}$. However, while they show that flow is dispensable for the initial formation of the DLAV, little is known about the cellular mechanism driving the anastomosis of ipsilateral ISVs and the lumenisation of the DLAV segments during DLAV formation (32 to $48 \mathrm{hpf}$ ). 
Recent reports have characterised the importance of svep1/polydom, a secreted ECM protein that has been reported to mediate cell-to-substrate adhesion in vitro, in an integrin $\alpha 9 \beta 1$-dependent manner [13], as a regulator of secondary angiogenesis [14, 15]. In zebrafish, loss of function svep1 mutants exhibit a reduced number of venous and lymphatic precursor (parachordal lymphangioblasts, PLs) emerging from the PCV during secondary angiogenesis (from $32 \mathrm{hpf}$ ). In addition, PLs show reduced migration capacity from the horizontal myoseptum. Both these defects lead to an increased number of arterial ISVs (aISVs) and a severe reduction in lymphatic trunk vasculature. Here, we uncover a completely new and distinct role for Svep1 in the regulation of DLAV formation under reduced flow conditions, acting in part through modulation of Vegfa/Vegfr signalling in endothelial cells.

\section{Material and Methods}

\section{Zebrafish husbandry and transgenic lines}

Zebrafish (Danio rerio) were raised and staged as previously described [16]. The following transgenic lines were used: Tg[fli1a:EGFPJy' [17] (labeling all endothelial cells), Tg[gata1a:dsRed]sd2 [18] (labeling all erythrocytes), $\operatorname{Tg}[-0.8 f l t 1: R F P]^{h u 5333}$ [5] (strongly labels arterial endothelial cells), TgBAC(apln:eGFP)bns157 [19] (labeling endothelial tip cells). Tg(svep1:Gal4FF;UAS:GFP) (labeling svep1 positive cells) [14].

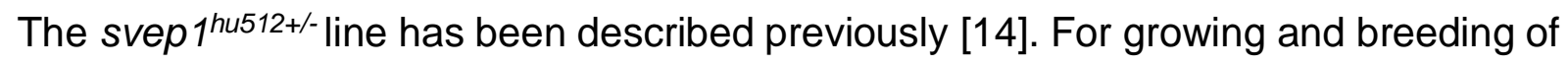
transgenic lines we comply with regulations of the ethical commission animal science of MDC Berlin and with FELASA guidelines [20].

\section{Tricaine treatment}


To slow down heart rate and blood flow during DLAV formation, embryos were treated with $0.007 \%, 0.014 \%(1 \mathrm{X})$ or $0.028 \%(2 \mathrm{X})$ tricaine (MS-222, Sigma) between 30 and $48 \mathrm{hpf}$, as indicated in the figure legends.

\section{Morpholino knockdown}

Morpholinos against svep1 (5ng) and flt1 (1ng) were used as previously described in $[15,21]$ and injected in the yolk of zebrafish embryos at the one-cell stage.

\section{Statistical Analysis}

All quantifications were performed in the trunk region of zebrafish embryos, across 7-

9 somites. $(\mathrm{N})$ refers to experimental replicates, $(\mathrm{n})$ refers to number of embryos. Statistical analysis was performed with Mann-Whitney-U tests, unless indicated otherwise. No statistical method was used to predetermine sample size. Data represent mean \pm standard deviation of representative experiments (except when indicated otherwise). Statistical tests were conducted using Prism (GraphPad) software. Adequate tests were chosen according to the data to fulfil test assumptions. Sample sizes, number of repeat experiments, performed tests and p-values are indicated per experiment.

Zebrafish embryos were selected based on the following pre-established criteria: normal morphology, beating heart, presence of circulating red blood cells. The experiments were not randomized. For every experiment treated and control embryos were derived from the same egg lay. The investigators were not blinded to allocation during experiments and outcome assessment.

\section{Live imaging}


Embryos were anaesthetized in $0.014 \%$ tricaine (MS-222, Sigma), mounted in a 35 $\mathrm{mm}$ glass bottom petri dish $(0.17 \mathrm{~mm}$, MatTek) using $0.6-1 \%$ reduced melting point agarose (Sigma) containing $0.014 \%$ tricaine, and bathed in E3 media containing $0.014 \%$ ( $1 \mathrm{X}$ tricaine) and $0.003 \%$ PTU. Time-lapse imaging was performed using an upright 3i spinning-disc confocal using a Zeiss Plan-Apochromat, 20x, 40x/1.0 NA water-dipping objective. Image processing was performed using Fiji software [22].

\section{Isolation of endothelial cells}

$48 \mathrm{hpf}$ Tg[fli1a: nEGFP ${ }^{77}$ and Tg[fli1a: EGFP ${ }^{y 1}$ crossed to Tg[gata1:dsRed] embryos were dechorionated using a solution of $1 \mathrm{mg} / \mathrm{mL}$ Pronase (Sigma Aldrich) on an orbital shaker for 10 minutes at room temperatures. Up to 250 dechorionated embryos per conditions were anaesthetized with $1 \mathrm{X}(0.014 \%)$ tricaine and transferred to a $1.5 \mathrm{~mL}$ Eppendorf with $1 \mathrm{~mL}$ calcium-free Ringer solution (116 mM NaCL, $2.9 \mathrm{mM} \mathrm{KCL}, 5 \mathrm{mM}$ HEPES pH7.2) to remove the yolks. After pipetting gently up and down with a $1 \mathrm{~mL}$ tip the embryos were centrifugated at $2000 \mathrm{rpm}$ for 5 minutes at $4^{\circ} \mathrm{C}$. The supernatant was removed, and the procedure repeated until all the yolks were removed and the solution clear. The calcium-free ringer solution was replaced with $1 \mathrm{~mL}$ of protease solution (72 $\mu \mathrm{g} / \mathrm{mL}$ Liberase $\mathrm{DH}$ research grade from Merck/Sigma, $0.4 \mathrm{U} / \mathrm{mL}$ DNaselInvitrogen). The embryos were incubated at $28.5 \mathrm{C}$ on an orbital shaker for 20 minutes, pipetting up and down with a $200 \mu \mathrm{L}$ tip every 3 minutes, to form a homogenous solution of cells. The dissociation process was stopped by placing the embryos on ice and adding $2 \mu \mathrm{L} \mathrm{CaCl} 2$ and $0.5 \mu \mathrm{L}$ FBS per $\mathrm{mL}$. The cell suspension was centrifuged for at $2000 \mathrm{rpm}$ for 5 minutes at $4^{\circ} \mathrm{C}$. The supernatant was discarded, and the cells resuspended in sorting solution (2mM EDTA, 0.4U/mL DNasel, 0.5\% FBS in DPBS). The solution was passed through a $40 \mu \mathrm{m}$ strainer inside a $50 \mathrm{~mL}$ falcon tube, previously washed with $500 \mu \mathrm{L}$ sorting solution. Following filtration, $500 \mu \mathrm{L}$ sorting 
solution was added to the strainer. The filtered solution was centrifuged at 2000 RPM for 5 minutes at $4 \mathrm{C}$. The supernatant was removed, and the cell resuspended in 700 $\mu \mathrm{L}$ sorting solution. The cell suspension was then loaded onto an ARIA III FACsorter (BD Bioscience). Using Tg[gata1:dsRED] only embryos for gating, we specifically sorted GFP+, dsRED- cells to remove red blood cells with fli1a promoter activation at this specific developmental stage. Upon centrifugation and removal of the supernatant, all cells were stored immediately at $-80 \mathrm{C}$ until protein extraction.

\section{Protein extraction}

Sorted embryos were treated with $40 \mu \mathrm{L}$ Lysis Buffer $(1 \mathrm{~mL} 1 \mathrm{M}$ Tris- $\mathrm{HCl}, 0.4 \mathrm{~mL}$ 0.5M EDTA, $8.75 \mathrm{~mL} 10 \%$ Brij 96, $1.25 \mathrm{~mL} 10 \% \mathrm{NP}-40$ to $100 \mathrm{~mL}$ with $\mathrm{dH} 2 \mathrm{O}$ ) and $0.4 \mu \mathrm{L}$ Protease Inhibitor cocktail (ThermoFischer). The samples were homogenized with a pestle and centrifuged at 13000 RPM for 15 minutes at $4 \mathrm{C}$. The protein supernatant was collected, and protein concentration assessed using a BCA protein assay kit (ThermoFischer).

\section{Western Blot}

$20-50 \mu \mathrm{g}$ of the protein lysates (equal amount for each condition to compare) were diluted in $18.7 \mu \mathrm{L}$ water and $6.3 \mu \mathrm{L}$ loading buffer $(25 \mu \mathrm{L}$ total volume) and heated for 5 min at $95^{\circ} \mathrm{C}$ to denature proteins. The samples were loaded and run alongside a $10 \mu \mathrm{l}$ ladder marker (Novex Sharp Pre-Stained - thermoFischer) for $1 \mathrm{~h}$ at $150 \mathrm{~V}$ and subsequently transferred onto previously $\mathrm{MeOH}$ activated polyvinylidene fluoride membranes. Membranes were blocked with $5 \%$ non-fat dry milk in $50 \mathrm{mg} / \mathrm{mL}$ TBS-T for $1.5 \mathrm{~h}$ at room temperature and then incubated with primary antibody against $\mathrm{p}-\mathrm{ERK}$, overnight at $4^{\circ} \mathrm{C}(1: 250$, (Erk1/2) (Thr202/Tyr204) \#9101, Cell Signaling Technology). 
After incubation with primary antibody, the membranes were washed 4 times in 50 $\mathrm{mg} / \mathrm{mL}$ TBS-T and then incubated with secondary antibodies (1:4000 anti-rabbit) and washed 3 times with $50 \mathrm{mg} / \mathrm{mLTBS}-\mathrm{T}$. Immunodetection was performed using a chemiluminescence kit (1mL SuperSignal West Dura; Pierce), and bands were developed using the Las-4000 imaging system. After initial immunodetection, membranes were stripped of antibodies by using the Stripping kit (ThermoFisher) at $56^{\circ} \mathrm{C}$ for $40 \mathrm{~min}$ and re-probed with anti-GFP antibody for $1 \mathrm{~h}$ (1:1000, Origene R1091P). Band intensity was measured using the histogram function on the Fiji software, with control and treated samples on the same blot [22].

\section{Blood flow and heart rate measurements}

Embryos were anaesthetized and imaged on an upright 3i spinning-disc confocal using a Zeiss Plan-Apochromat, 20x/1.0 NA water-dipping objective with a frame interval of $10 \mathrm{~ms}$. Kymographs were generated using the MultipleKymograph plugin in ImageJ to quantify heart rate over an 8 second period, synced to the beginning of a heartbeat (line width: 1).

To estimate instantaneous blood flow speed, we cropped images of the dorsal aorta and measured average frame-to-frame translation of red blood cells using the KuglinHines algorithm (Kuglin and Hines, 1975) for image phase-correlation. In brief, the phase correlation map between two adjacent frames was calculated by multiplying the Fast Fourier transform (FFt) of frame $i$ and a conjugate FFt of frame ${ }_{i+1}$. The inverse FFt of the phase correlation provides a correlation map with a peak offset from the center by the relative shift between the frames. The position of the peak was determined by finding the local maximum in a Gaussian filtered correlation map. The velocity data 
was smoothed with a moving average filter with a span of 5 frames. Analysis was performed in Matlab (Mathworks, Inc.).

\section{Chemical treatment}

Where indicated, embryos were treated with VEGFR inhibitors ZM323881 (Tocris Bioscience) or SU5416 (sigma Aldrich), from 30 to $48 \mathrm{hpf}$, in addition to $0.003 \%$ PTU and the indicated amount of tricaine.

\section{Results}

svep1 mutant and morphant zebrafish embryos exhibit vascular anastomosis defects. 
Imaging angiogenesis in the trunk of svep1 loss-of-function mutants (Ly02-12 - named

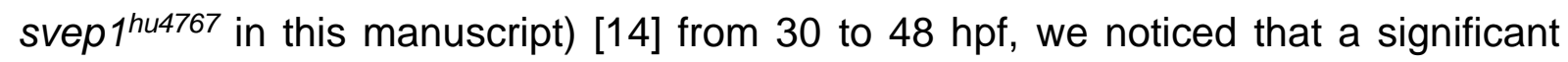
number of primary angiogenic sprouts failed to anastomose with their ipsilateral neighbours (Figure 1A) (Supplementary Video 1,2$)$. In the majority of cases $(74 \% \pm$ 33), the DLAV gaps arise following the regression of a pre-existing connection between ipsilateral neighbouring ISVs, rather than an absence of connection ( $\mathrm{N}=6$ experiments, $\mathrm{n}=18$ mutants). Additionally, only a minority of these connections (13\%, N=6 experiments, $n=17$ mutants) were transiently lumenised before regressing. These results suggest that in this context, svep1 loss of function negatively affects the stabilisation of vascular connections between neighbouring sprouts.

We quantified the number of gaps in the DLAV, and lumenisation status of existing DLAV segments at $48 \mathrm{hpf}$, a time at which the DLAV is considered fully formed and almost fully lumenised in wild type (WT) zebrafish embryos [2, 12]. svep $1^{512}$ mutants exhibited a significantly increased number of gaps in their DLAV at $48 \mathrm{hpf}(25.5 \% \pm$ 23.4 versus $2.6 \% \pm 5.6$ in their WT siblings) and a significant decrease of lumenised DLAV segments $(47.8 \% \pm 33.1$ versus $86.4 \% \pm 17.3)$ (Figure 1B-D). These phenotypes were also observed in svep1 morphants compared to control morphants (DLAV gaps: $36.3 \% \pm 25.4$ versus $6 \% \pm 10.9$; lumenised DLAV segments: $46.1 \% \pm$ 33.8 versus $94.7 \pm 10.5$ ) (Figure $1 E, F$ ). In addition, while the expressivity of the morphant phenotype in different zebrafish transgenic lines and clutches varied markedly, we observed a statistically significant difference between svep 1 and control morphants in all cases, (Supplementary Figure 1). 
Surprisingly, we could not detect any anastomosis defects at the DLAV in svep $1^{512}$ mutants and morphants when imaged at $2 \mathrm{dpf}$, whilst they continued to exhibit the previously reported PL phenotypes [14] (PLs at the horizontal myoseptum: $47.6 \% \pm$ 17.5 versus $80.2 \% \pm 19.4 \%$ in WT clutch mates and alSVs: $65.4 \% \pm 15.1$ versus $49.3 \%$ \pm 15 in WT siblings, $\mathrm{N}=4, \mathrm{n}=12$ mutants, $\mathrm{n}=25 \mathrm{WT}))$. The DLAV phenotypes instead only occurred in mutant embryos that were live imaged from 30 to $48 \mathrm{hpf}$. Upon closer inspection, we found that treatment with tricaine (tricaine mesylate - MS222), a muscle relaxant commonly used to immobilise zebrafish embryos, lead to a dose-dependent emergence of the DLAV phenotypes in svep1 loss-of-function morphants (Figure 2A, B) following treatment with concentration of $1 \mathrm{X}(0.014 \%)$ or above from 30 to $48 \mathrm{hpf}$. In addition, removal of tricaine from mutant embryos lead to a significant recovery of the DLAV vasculature at $72 \mathrm{hpf}$, with only $27 \%( \pm 26)$ of the DLAV gaps still present at that stage (supplementary Figure 2A, B).

Previous work revealed that svep1 loss of function is associated with cardiac defects [23]. To test if svep1 loss-of-function augments tricaine-induced blood flow reduction, we quantified heartbeats per minutes in control and svep1 morphants and found no differences in embryos treated with $1 \mathrm{X}(0.014 \%)$ or $2 \mathrm{X}(0.028 \%)$ tricaine from 30 to 48 hpf (supplementary Figure 2C). However, we found the mean blood flow speed to be significantly decreased in svep1 morphants compared to control morphants when treated with $1 X$ tricaine (MO-CTL 5ng: 679um/s \pm 205, MO-svep1 5ng: $521 \mathrm{um} / \mathrm{s} \pm$ 190). This suggests that the cardiac phenotype reported in svep1 loss-of-function embryos exacerbates the blood flow reduction induced by tricaine treatment. 
To further characterize the phenotype, we therefore decided to begin our investigation at $30 \mathrm{hpf}$, as this time point marks the beginning of ISV ipsilateral anastomosis in most embryos (Figure 1A). As tricaine treatment leads to a reduction of blood flow speed [24], we investigated whether a general blood flow speed reduction or a reduction of erythrocytes-dependent shear stress is responsible for the DLAV phenotype in svep1 morphants. In the absence of tricaine treatment, simultaneous inhibition of svep1 function and erythrocyte formation, using svep1 and gata1 morpholinos, did not lead to any DLAV phenotypes (Figure 2C, D), suggesting that shear stress does not modulate DLAV formation at that developmental stage. However, in the absence of tricaine treatment, complete abolition of blood flow using the tnnt2a morpholino lead to a DLAV phenotype in svep1 morphants (Figure 2E, F) $(16.4 \% \pm 16.2$ versus $3.5 \pm 6.2$ in svep1 morphants only). Abolition of blood flow in svep1 homozygous mutants further increases the proportion of fish exhibiting a strong DLAV phenotype (30\% compared to $5.3 \%$ of WT clutch mate) (Supplementary Figure $2 \mathrm{~F}$ ). These results suggest that while svep1 loss-of-function produces a cardiac defect that enhances the effect of tricaine on reducing blood flow, svep1 has an additive effect in modulating blood vessels anastomosis.

Finally, we observed that svep 1 loss of function significantly increases the percentage of short ISVs in tnn2a morphants at $48 \mathrm{hpf}(14.6 \% \pm 10.5$ versus $4.8 \% \pm 7.6$ in tnnt2a morphants only). This led us to investigate the importance of svep1 in the regulation of angiogenic sprout identity and behaviour under reduced flow conditions.

Svep1 is expressed in neurons of the neural tube and its expression is flow dependent 
Imaging of the svep1 reporter line Tg(svep1: Gal4FF;UAS:GFP) at $48 \mathrm{hpf}$ showed strong GFP expression in dorsal epithelial cells, above the neural tube, and in individual neurons of the neural tube (Figure 3A, Supplementary Figure 3A, B). Treatment with $1 \mathrm{X}$ tricaine from 30 to $48 \mathrm{hpf}$ led to a significant reduction in svep1 expression throughout the trunk area, particularly in the neural tube at $48 \mathrm{hpf}$ (Figure 3B). In addition, tricaine treatment between 30 and $48 \mathrm{hpf}$ lead to a significant reduction in svep1 endogenous expression within the neural tube and ventral somite boundary (supplementary Figure 3C), suggesting that blood flow not only sensitises angiogenic sprouts to svep1 downregulation but also directly affects svep1 expression.

\section{svep1 loss-of-function leads to a defect in tip/stalk cell specification in primary angiogenic sprouts.}

The formation of the DLAV is initiated by the anastomosis of ipsilateral arterial sprouts, led by a tip cell [1]. To investigate tip cell identity in the zebrafish trunk, we took advantage of the recently published $\operatorname{Tg}(a p / n: e G F P)$ [19] reporter line, in which the nucleus of endothelial tip cells is highlighted by eGFP expression at $48 \mathrm{hpf}$. Following treatment with tricaine from 30 to $48 \mathrm{hpf}$, svep 1 morphants exhibited an expansion of Apln positive endothelial cells in intersegmental vessels (ISVs), while control morphants predominantly presented Apln positive cells in the dorsal-most region of the vasculature, consistent with a contribution of tip cells to the formation of the DLAV (Figure 3C-F). In addition, svep1 morphants presented with an overall increase in Apln positive cells per ISVs $(56.5 \% \pm 24$ versus $17.8 \% \pm 13.3$ in control morphants)(Figure $3 G)$. 
As tip cell identity is in part characterised by increased levels of $p$-ERK downstream of Vegfa/Vegfr signalling, we next investigated this pathway activation in svep1 morphants. Interestingly, sorted endothelial cells from $48 \mathrm{hpf}$ svep 1 morphants treated with $1 \mathrm{X}$ tricaine from $30 \mathrm{hpf}$, did not show a significant increase in p-ERK levels normalised to total ERK levels (Figure $3 \mathrm{H}, \mathrm{I}$ ). Overall, these results suggest that the DLAV phenotype present in svep1 morphants treated with tricaine is associated with a defect in tip/stalk cell specification in primary angiogenic sprouts.

To test whether the observed expansion of tip cell specification is caused by the augmented reduction in blood flow speed observed in svep 1 loss-of-function embryos, we investigated tip cell identity in control embryos treated with $2 \mathrm{X}$ tricaine compared to $1 \mathrm{X}$ tricaine. $2 \mathrm{X}$ tricaine treatment alone results in an exacerbated blood flow reduction to that observed in svep 1 loss-of-function embryos treated with $1 \mathrm{X}$ tricaine (451 um/s \pm 231.6 , as presented by our laboratory in a recent manuscript [24], versus $521 \mathrm{um} / \mathrm{s} \pm 190)$. However, treatment with $2 \mathrm{X}$ tricaine of Apln-GFP embryos did not cause expansion of tip cell specification (Figure $3 \mathrm{~J}, \mathrm{~K}$ ), suggesting that this phenotype is primarily a consequence of svep1 loss-of-function under reduced flow condition.

svep1 loss-of-function and knockdown are rescued by flt1 knockdown.

To investigate whether the tip cell phenotype is mediated by, or dependent on, increased Vegfa/Vegfr signaling in svep1 morphants, we decided to modulate it in vivo, first by targeting Flt1 expression. In mice and zebrafish, Flt1 mainly functions as a decoy receptor with high affinity for Vegfa during development to modulate the activation of the Vegfa/Vegfr signalling pathway [21, 25, 26]. Alternative splicing of flt1 generates two isoforms: a membrane bound form (mFlt1), and a soluble form (sflt1, an 
alternative spliced and secreted form of mFlt1) [27]. Reports have shown that sFlt1 acts as a negative regulator of tip cell formation in the zebrafish trunk [28].

To reduce flt1 expression, we used a morpholino targeting both mFlt1 and sFlt1 expression. Similar to previous observations [21], flt1 morphants do not exhibit any DLAV defects at $48 \mathrm{hpf}$ when treated with tricaine from 30 to $48 \mathrm{hpf}$. However, in svep1 mutants and morphants, knockdown of flt1 expression lead to a rescue of DLAV formation defects (Figure 4A, B). Interestingly, flt1 knockdown rescued the DLAV segment lumenisation phenotype only in svep1 morphants but not in mutant embryos (Figure 4C, D), suggesting potential differences in the expressivity of the flt1 knockdown in svep1 mutant and morphants.

In addition to the DLAV rescue, flt1/svep1 double knockdown led to the formation of aberrant arterial loops in alSVs $(18.5 \% \pm 12.4$ versus $0.8 \% \pm 2.7$ in $M O-s v e p 1$ and $3.6 \% \pm 6.1$ in $M O$-flt1 only) (Figure 4E, F). The majority of these arterial loops were lumenised and composed of more than one endothelial cell ( $>1$ cell/loop: $94.1 \%$ in $\mathrm{n}=34$ loops). In 9.3\% of somites (26/280), we also observed abnormal alSVs to alSVs connections (Figure 4H), which were never seen in control embryos. Thus, the rescue of connectivity at the DLAV level through flt1 knockdown was accompanied by an excess connectivity at aberrant locations.

Importantly, flt1 morphants treated with $2 \mathrm{X}$ tricaine from 30-48 hpf exhibited aberrant arterial loops in only $2.4 \% \pm 4.1$ alSVs, while control morphants under the same treatment never exhibited any $(\mathrm{N}=3, \mathrm{n}=21 \mathrm{MO}-\mathrm{flt} 1 \mathrm{ng}, \mathrm{n}=24 \mathrm{MO}-\mathrm{CTL}$ 1ng). In both conditions, we could not find abnormal aISVs to alSVs connections. These results 
suggest that svep1 loss-of-function, rather than reduced blood flow, is the principal driver for the excess connectivity observed with concomitant flt1 knockdown.

In search for the underlying cause of this hyperconnectivity, we investigated tip cell specification using the Apln-GFP transgenic reporter line. In embryos treated with $1 \mathrm{X}$ tricaine from 30 to $48 \mathrm{hpf}$, flt 1 knockdown led to an expansion and increase of the total number of Apln+ endothelial cells in alSVs, despite no significant DLAV formation defects (Figure 4I-K). svep1/fIt1 double morphants exhibit significantly more Apln+ endothelial cells in the dorsal and ventral part of aISVs, and more Apln+ cells per alSV than both svep1 and flt1 morphants alone (more than one Apln+ cell in $87.6 \% \pm 13.9$ ISVs versus $70.3 \% \pm 17.7$ in svep1 only morphants and $63.9 \pm 21.9$ in flt 1 only morphants)(Figure 1L).

These results suggest that the expansion of the number of tip cells in alSVs per se is not the driver of anastomosis defects.

\section{Vegfa/Vegfr signalling is necessary for ISV lumenisation maintenance and DLAV formation}

Vegfa/Vegfr signalling regulates primary angiogenic sprouting in the developing zebrafish trunk. Inhibition of Vegfr tyrosine kinase activation between 18 and 20 hpf results in the absence of angiogenic sprouting from the dorsal aorta [29]. However, to our knowledge, there exist no reports for the role of active Vegfa/Vegfr signalling in the initial formation and lumenisation of the DLAV (30-48 hpf). As a polarised increase in Vegfa/Vegfr signalling is necessary to establish tip and stalk cells identity in the growing sprouts [6] and a local increase in VEGFA signalling is essential for the 
establishment of stable connections between vascular sprouts in vivo and in vitro [30], we decided to investigate the effect of a general reduction of this signalling pathway on the formation of a lumenised DLAV. For this purpose, we used the VEGFR2 inhibitor ZM323881, a tyrosine-kinase inhibitor that reduces Vegfa/Vegfr signalling [31], confirmed by down-regulation of $p$-ERK in FAC sorted endothelial cells from embryos treated with 50 nM ZM323881 and 1X tricaine from 30 to 48 hpf (Supplementary Figure 3A-B). Consistent with its function, ZM323881-mediated down regulation of Vegfa/Vegfr signalling can be partially rescued with down-regulation of flt 1 expression (Supplementary Figure 3E, F).

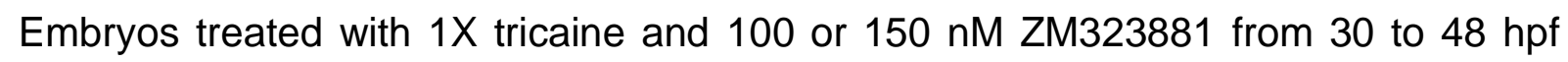
exhibited significant DLAV defects (Supplementary Figure 3C, D). In addition, even at the highest concentration not inducing any significant defect in DLAV establishment (50nM), we observed a strong defect in ISV lumenisation at $48 \mathrm{hpf}$ (Figure 5D), suggesting that in addition to its importance in the ipsilateral anastomosis of alSVs and DLAV lumenisation, Vegfa/Vegfr signalling is important for the maintenance of alSV lumenisation.

\section{Vegfa/Vegfr signalling inhibition exacerbates svep1 loss-of-function DLAV phenotype in reduced flow conditions.}

As the DLAV phenotype in svep1 morphants can be rescued by an increase in Vegfa/Vegfr signalling (Figure 3), and because an expansion of tip cell numbers within alSVs does not appear to be causative for ipsilateral anastomosis defects (Figure 4), we investigated whether reducing Vegfa/Vegfr signalling in svep1 morphants would exacerbate the DLAV defect observed under reduced flow conditions. 
We took advantage of the variability in expressivity of the DLAV phenotype observed in different reporter lines (Supplementary Figure 1) to select one presenting with a limited DLAV phenotype when injected with svep1 morpholino and treated with $1 \mathrm{X}$ tricaine from 30 to $48 \mathrm{hpf}$. In this context, we find that concomitant treatment with 50 nM ZM323881 results in a significant increase in the number of gaps in the DLAV $(20.3 \% \pm 21.8$ versus $5.4 \% \pm 9.3)$ and in a reduction of the number of lumenised DLAV segments (35.4 \pm 33.9 versus $76.9 \pm 24.9$ ) (Figure $5 A-C)$. Supporting this notion, we found that treatment with the commonly used VEGFR signalling inhibitor SU5416 [3, $12,32,33$ ] also exacerbated the DLAV phenotype in svep1 morphants treated with $1 \mathrm{X}$ tricaine from 30 to $48 \mathrm{hpf}$ (supplementary Figure 5A-C).

In addition, we observed that svep1 morphants exhibited a mild but significant reduction in ISV lumenisation that was increased by treatment with $50 \mathrm{nM}$ ZM323881 under $1 \mathrm{X}$ tricaine conditions $(26.9 \% \pm 22.6$ versus $80.3 \% \pm 16.5)$ ) (Figure 5E). Furthermore, we found that concomitant svep1 knockdown and Vegfa/Vegfr signalling inhibition lead to the emergence of embryos with a significant number of ISVs missing

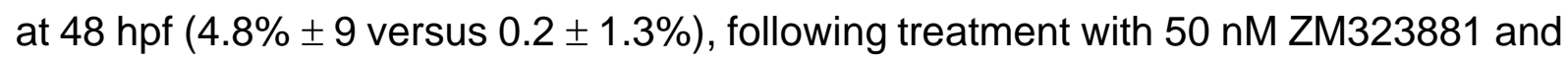
$1 \mathrm{X}$ tricaine from 30 to $48 \mathrm{hpf}$ (Figure 5F). Finally, we observed that in the context of Vegfr signalling inhibition, further reducing blood flow with increased tricaine concentration (2X) inhibition did not result in the same phenotypic severity as in svep1 loss-of-function embryos treated with $1 \mathrm{X}$ tricaine. In embryos treated with 50nM ZM323881 and $2 X$ tricaine from 30 to $48 \mathrm{hpf}, 40.9 \% \pm 21$ of ISVs are lumenised (compared to $26.9 \% \pm 22.6$ in mild svep 1 morphants treated with $1 \mathrm{X}$ tricaine and $50 \mathrm{nM}$ Zm323881) and $3.3 \% \pm 5.8$ of ISVs are missing at $48 \mathrm{hpf}$ (compared to $26.9 \% \pm 22.6$ 
in mild svep1 morphants treated with $1 \mathrm{X}$ tricaine and 50nM Zm323881). This suggests that reduced function or loss of Svep1 and reduced blood flow both contribute to the emergence of a vascular phenotype in the context of Vegfa/Vegfr signalling inhibition (Figure 5G-J).

Overall, these results suggest that Svep1 and Vegfa/Vegfr signalling appear to act synergistically to maintain vessel lumenisation and stability under low flow conditions.

\section{Discussion}

The question of how vessels anastomose remains incompletely understood, given the complexities of endogenous and exogenous signals driving vascular remodelling and development in parallel and sometimes synergistic ways [1]. Careful and granular analysis of early stage angiogenesis has shed light on the temporal and morphological dynamics underlying this process $[11,34,35]$. Following the establishment of a stable connection between neighbouring tip cells, supported by the local deposition of adherens junctions proteins, such as VE-cadherin, F-actin and ZO-1 [35, 36], ISV connections become progressively lumenised. Lumenisation and stabilisation of ISV connections is thought to occur either through a flow-dependent transcellular hollowing of connecting tip cells (Type I anastomosis)[35, 37] or through a flow-independent process involving the coalescence of isolated luminal pockets into a single luminal space that will subsequently be perfused (Type II anastomosis)[34]. However, comparatively little is known about the molecular pathways leading to the formation and stabilisation of nascent anastomotic connections. 
Here, we identified blood flow and Svep1 as regulators of vessel anastomosis in the developing zebrafish vasculature. Both appear to play a role in the stabilisation of nascent anastomotic connections between neighbouring vessels. Under reduced flow conditions, svep1 knockdown or loss-of-function scenarios result in reduced anastomosis of ipsilateral ISVs and defective formation of a lumenised DLAV at 48 hpf. We find that endothelial cells in svep1 morphants do not show significantly increased Vegfa/Vegfr signalling. This is surprising given the concomitant increase in Apelin positive cells in ISVs, as tip cells specification has been shown to correlate with higher level of Vegfa/Vegfr signaling [38]. We can speculate that the increase of Vegfa/Vegfr signalling in the trunk ISV of svep1 morphants is not ubiquitous across the endothelium and therefore cannot be detected with a global endothelial analysis. Alternatively, the increase and expansion of Apln levels in tip cells could be independent of increased ERK activity or Vegfr signalling.

However, neither a Vegfa/Vegr signalling modulation nor the increased tip cell numbers appear causative, as the DLAV defect can be rescued with flt1 knockdown and is exacerbated by Vegfa/Vegfr signalling inhibition. Interestingly, while further inhibition of blood flow speed below that exhibited in a context of svep 1 loss-of-function and $1 \mathrm{X}$ tricaine treatment does similarly result in visible anastomosis defect in the DLAV region, these are not associated with a significant increase of Apelin positive cells in the trunk ISV, supporting our hypothesis that the expansion of the number of tip cells in alSVs per se is not the driver of anastomosis defects.

The results about the role of Vegfa/Vegfr signalling in mediating vessel anastomosis in the zebrafish trunk appear at first sight hard to reconcile. However, previous work 
on the role of VEGFR2 signalling in anastomosis allow us to speculate on the molecular mechanisms at play here.

In the mouse retina, Nesmith and colleagues have shown that endothelial cells presenting with reduced Flt1 expression were more likely to form stable connections with approaching sprouts [30]. They also clarified that it is reduced mFlt1 expression that influences the bias towards stable connections, suggesting a cell-autonomous regulation of tip cell anastomosis. Finally, they remarked that sprouts with reduced Flt1 expression exhibit reduced exploratory transient connections with adjacent sprouts, proposing that this might lead to an increase in the number of suboptimal new vascular connections. We noticed a significant increase of vascular loops within ISVs, which can functionally be considered suboptimal or redundant for perfusion of tissues, in embryos with reduced svep1 and flt1 expression compared to flt1 knockdown alone. On its own, this result suggests that Svep1 role in vascular development might occur through the modulation of Flt1 activity in vivo. As the function of Flt1 in developmental angiogenesis appears strictly limited to its role as a decoy receptor for VEGFA [26, 39], we can speculate that Svep1 might support/enhance Flt1 decoy ability. Alternatively, we can speculate that flt1 and svep 1 loss-of-function both drive excess tip cell formation in an additive fashion, and that the DLAV rescue observed in embryos with both loss-of-function could potentially result from an increased tip cell activity, driven by Vegfa/Vegfr signalling increase. This hypothesis would reconcile with our observation that in the context of reduced blood flow, in which embryos present with DLAV formation defects without increase in tip cell numbers, Vegfa/Vegfr signalling increase results in a rescue of the DLAV phenotype without aberrant ISV connections. 
However, under reduced flow conditions, while flt1 knockdown enhances the formation of stable connections between ipsilateral neighbouring sprouts, we find that svep1 knockdown alone leads to a significant reduction in the stability of these connections. In addition, we find that inhibition of Vegfa/Vegfr signalling leads to a DLAV phenotype comparable to that observed in svep1 mutant and morphants, and that Vegfa/Vegfr inhibition in svep1 morphants leads to a strong increase of anastomosis defects. Taken together, these results suggest that any potential increase in Vegfa/Vegfr signalling following svep1 knockdown would be compensatory in nature and insufficient to stabilise new connections in the absence of flow.

The importance of blood flow inhibition in the emergence of the DLAV phenotype might suggest a potential investigative avenue. In embryos with reduced blood flow and concomitant reduced Svep1 levels or reduced Vegfa/Vegfr signaling, we observed a significant reduction in ISV lumenisation. This suggest that in this context, a significant proportion of ISVs might initiate anastomosis through flow-independent pathways (Type II anastomosis). We can speculate that blood flow plays a positive role in the stabilization of type II anastomosis between neighboring ISVs, which would explain the DLAV formation defect observed in reduced (tricaine treatment) or abolished (tnnt2a morpholino) blood flow conditions. The significant increase in DLAV phenotype observed in svep1 loss-of-function and abolished flow conditions suggest a role of Svep1 in regulating vessel anastomosis specifically under reduced flow conditions.

In addition, while blood flow reduction between 30 and 48 hpf is sufficient to induce anastomotic defects in the DLAV, this phenotype is significantly exacerbated with 
concomitant treatment with Vegfr signalling inhibitors, supporting the idea that flow and Vegfa/Vegfr signaling act synergistically to support vessel anastomosis.

Svep1(also known as Polydom) is a secreted ECM protein that has been reported to mediate cell to substrate adhesion in vitro, at leasr in part in an integrin $\alpha 9 \beta 1$ dependent manner [13]. Although integrin $\alpha 9$ zebrafish mutants fail to display a vascular phenotype other than lymphatic valve formation [40], interaction with another member of the Integrin family could prove more relevant. For example, Integrin $\beta 1 b$ appears to be important for the formation of the DLAV in zebrafish embryos [41]. Future efforts in identifying interaction partners for Svep1 will further enhance our understanding of the molecular pathways regulating vessel anastomosis. Imaging of the svep1 reporter line $T g($ svep1: Gal4FF;UAS:GFP) at 48 hpf showed strong GFP expression in dorsal epithelial cells, above the neural tube, and in individual neurons of the neural tube in close proximity to the anastomotic bridges forming between adjacent ISVs. We can speculate that neuronal expression of Svep1 is what locally regulates vessel anastomosis, in a non-cell autonomous manner. In addition, Svep1 is a multi-domain protein, and the importance of individual domains could be functionally tested in reduced flow conditions, by the generation of zebrafish lines expressing selective truncated forms of Svep1. Finally, the significant differences observed in svep1 loss-of-function phenotype expressivity between zebrafish lines might offer an interesting avenue to decipher the compensatory mechanisms at play and reveal new molecular pathways interacting with Svep1 in the regulation of vessel anastomosis.

Understanding the genetic regulation of phenotypic robustness in angiogenesis and its failure [42] promises crucial insights into the mechanisms causing breakdown of vascular homeostasis in human disease. 


\section{Acknowledgments}

We thank all members of the Gerhardt lab for interesting discussions and comments, as well as the Zebrafish facility staff at the MDC for excellent animal care.

\section{Source of Funding}

This work was supported by the DZHK (German Centre for Cardiovascular Research).

B. Coxam was supported by a DZHK excellence Grant (Postdoc Start-up Grant - EX2-

B DR_Coxam). This project and was supported by a grant from the Fondation Leducq (17 CVD 03) and from the DFG (CRC1348; Y.P. and S.S.-M.). We thank members of the Gerhardt lab for fruitful discussions and comments.

\section{Disclosure}

None

\section{References:}

1. Hogan, B.M. and S. Schulte-Merker, How to Plumb a Pisces: Understanding Vascular Development and Disease Using Zebrafish Embryos. Dev Cell, 2017. 42(6): p. 567-583.

2. Isogai, S., et al., Angiogenic network formation in the developing vertebrate trunk. Development, 2003. 130(21): p. 5281-90.

3. Covassin, L.D., et al., Distinct genetic interactions between multiple Vegf receptors are required for development of different blood vessel types in zebrafish. Proc Natl Acad Sci U S A, 2006. 103(17): p. 6554-9.

4. Covassin, L.D., et al., A genetic screen for vascular mutants in zebrafish reveals dynamic roles for Vegf/Plcg1 signaling during artery development. Dev Biol, 2009. 329(2): p. 212-26.

5. Bussmann, J., et al., Arteries provide essential guidance cues for lymphatic endothelial cells in the zebrafish trunk. Development, 2010. 137(16): p. 2653-7.

6. Shin, M., et al., Vegfa signals through ERK to promote angiogenesis, but not artery differentiation. Development, 2016. 143(20): p. 3796-3805.

7. Bahary, N., et al., Duplicate VegfA genes and orthologues of the KDR receptor tyrosine kinase family mediate vascular development in the zebrafish. Blood, 2007. 110(10): p. 3627-36. 
8. Bussmann, J., et al., Zebrafish VEGF receptors: a guideline to nomenclature. PLoS Genet, 2008. 4(5): p. e1000064.

9. Geudens, I. and H. Gerhardt, Coordinating cell behaviour during blood vessel formation. Development, 2011. 138(21): p. 4569-83.

10. Siekmann, A.F. and N.D. Lawson, Notch signalling limits angiogenic cell behaviour in developing zebrafish arteries. Nature, 2007. 445(7129): p. 781-4.

11. Betz, C., et al., Cell behaviors and dynamics during angiogenesis. Development, 2016. 143(13): p. 2249-60.

12. Zygmunt, T., et al., 'In parallel' interconnectivity of the dorsal longitudinal anastomotic vessels requires both VEGF signaling and circulatory flow. J Cell Sci, 2012. 125(Pt 21): p. 5159-67.

13. Sato-Nishiuchi, R., et al., Polydom/SVEP1 is a ligand for integrin alpha9beta1. J Biol Chem, 2012. 287(30): p. 25615-30.

14. Karpanen, T., et al., An Evolutionarily Conserved Role for Polydom/Svep1 During Lymphatic Vessel Formation. Circ Res, 2017. 120(8): p. 1263-1275.

15. Morooka, N., et al., Polydom Is an Extracellular Matrix Protein Involved in Lymphatic Vessel Remodeling. Circ Res, 2017. 120(8): p. 1276-1288.

16. Kimmel, C.B., et al., Stages of embryonic development of the zebrafish. Developmental dynamics : an official publication of the American Association of Anatomists, 1995. 203(3): p. 253-310.

17. Lawson, N.D. and B.M. Weinstein, In vivo imaging of embryonic vascular development using transgenic zebrafish. Dev Biol, 2002. 248(2): p. 307-18.

18. Traver, D., et al., Transplantation and in vivo imaging of multilineage engraftment in zebrafish bloodless mutants. Nature immunology, 2003. 4(12): p. 1238-46.

19. Marin-Juez, R., et al., Coronary Revascularization During Heart Regeneration Is Regulated by Epicardial and Endocardial Cues and Forms a Scaffold for Cardiomyocyte Repopulation. Dev Cell, 2019. 51(4): p. 503-515 e4.

20. Alestrom, P., et al., Zebrafish: Housing and husbandry recommendations. Lab Anim, 2019: p. 23677219869037.

21. Wild, R., et al., Neuronal sFlt1 and Vegfaa determine venous sprouting and spinal cord vascularization. Nat Commun, 2017. 8: p. 13991.

22. Schindelin, J., et al., Fiji: an open-source platform for biological-image analysis. Nat Methods, 2012. 9(7): p. 676-82.

23. Karpanen, T. and J. Olweus, The Potential of Donor T-Cell Repertoires in NeoantigenTargeted Cancer Immunotherapy. Front Immunol, 2017. 8: p. 1718.

24. Geudens, I., et al., Artery-vein specification in the zebrafish trunk is pre-patterned by heterogeneous Notch activity and balanced by flow-mediated fine-tuning.

Development, 2019. 146(16).

25. Ito, N., et al., Identification of vascular endothelial growth factor receptor-1 tyrosine phosphorylation sites and binding of SH2 domain-containing molecules. J Biol Chem, 1998. 273(36): p. 23410-8.

26. Hiratsuka, S., et al., Flt-1 lacking the tyrosine kinase domain is sufficient for normal development and angiogenesis in mice. Proc Natl Acad Sci U S A, 1998. 95(16): p. 9349-54.

27. Kendall, R.L. and K.A. Thomas, Inhibition of vascular endothelial cell growth factor activity by an endogenously encoded soluble receptor. Proc Natl Acad Sci U S A, 1993. 90(22): p. 10705-9. 
28. Krueger, J., et al., Flt1 acts as a negative regulator of tip cell formation and branching morphogenesis in the zebrafish embryo. Development, 2011. 138(10): p. 2111-20.

29. Fish, J.E., et al., Dynamic regulation of VEGF-inducible genes by an ERK/ERG/p300 transcriptional network. Development, 2017. 144(13): p. 2428-2444.

30. Nesmith, J.E., et al., Blood vessel anastomosis is spatially regulated by Flt1 during angiogenesis. Development, 2017. 144(5): p. 889-896.

31. Whittles, C.E., et al., ZM323881, a novel inhibitor of vascular endothelial growth factor-receptor-2 tyrosine kinase activity. Microcirculation, 2002. 9(6): p. 513-22.

32. Fong, T.A., et al., SU5416 is a potent and selective inhibitor of the vascular endothelial growth factor receptor (Flk-1/KDR) that inhibits tyrosine kinase catalysis, tumor vascularization, and growth of multiple tumor types. Cancer Res, 1999. 59(1): p. 99106.

33. De Angelis, J.E., et al., Tmem2 Regulates Embryonic Vegf Signaling by Controlling Hyaluronic Acid Turnover. Dev Cell, 2017. 40(4): p. 421.

34. Herwig, L., et al., Distinct cellular mechanisms of blood vessel fusion in the zebrafish embryo. Curr Biol, 2011. 21(22): p. 1942-8.

35. Lenard, A., et al., In vivo analysis reveals a highly stereotypic morphogenetic pathway of vascular anastomosis. Dev Cell, 2013. 25(5): p. 492-506.

36. Phng, L.K., F. Stanchi, and H. Gerhardt, Filopodia are dispensable for endothelial tip cell guidance. Development, 2013. 140(19): p. 4031-40.

37. Gebala, V., et al., Blood flow drives lumen formation by inverse membrane blebbing during angiogenesis in vivo. Nat Cell Biol, 2016. 18(4): p. 443-50.

38. Gerhardt, H., et al., VEGF guides angiogenic sprouting utilizing endothelial tip cell filopodia. J Cell Biol, 2003. 161(6): p. 1163-77.

39. Fong, G.H., et al., Role of the Flt-1 receptor tyrosine kinase in regulating the assembly of vascular endothelium. Nature, 1995. 376(6535): p. 66-70.

40. Shin, M., et al., Valves Are a Conserved Feature of the Zebrafish Lymphatic System. Dev Cell, 2019. 51(3): p. 374-386 e5.

41. lida, A., et al., Integrin beta1 activity is required for cardiovascular formation in zebrafish. Genes Cells, 2018. 23(11): p. 938-951.

42. Kasper, D.M., et al., MicroRNAs Establish Uniform Traits during the Architecture of Vertebrate Embryos. Dev Cell, 2017. 40(6): p. 552-565 e5. 


\section{Figure legends}

\section{Figure 1 - svep1 mutants and morphants zebrafish embryos exhibit vascular anastomosis defects.}

(A) Stills from time lapse movie of MO-CTL (5ng) and MO-svep1 (5ng) Tg(0.8flt1:RFP)huз3з3; TgBAC(flt4:Citrine) embryos treated with 1X $(0.014 \%)$ tricaine from 30 to $48 \mathrm{hpf}$. White asterisks indicate gaps in the DLAV.

(B) Still from a time lapse movie of MO-CTL (5ng) $T g(-0.8 f l t 1: R F P)^{\text {hu3333; }}$ $\operatorname{TgBAC}(f \mid t 4:$ Citrine) embryo exhibiting a gap in the DLAV between two adjacent ISVs. Side view, dorsal side left.

(C) Bilateral quantifications of the percentage of gaps in the DLAV at $48 \mathrm{hpf}$ in svep $1^{512}$ WT $(n=5)$, heterozygous $(n=18)$ and homozygous mutants $(n=10)$ treated with $1 \mathrm{X}$ tricaine $(0.014 \%)$ from 30 to $48 \mathrm{hpf}(\mathrm{N}=3)$.

(D) Bilateral quantifications of the percentage of lumenised segments in the DLAV at $48 \mathrm{hpf}$ in svep $1^{512}$ WT $(\mathrm{n}=5)$, heterozygous $(\mathrm{n}=18)$ and homozygous mutants $(n=10)$ treated with $1 X$ tricaine $(0.014 \%)$ from 30 to $48 \mathrm{hpf}(\mathrm{N}=3)$.

(E) Bilateral quantifications of the percentage of gaps in the DLAV at $48 \mathrm{hpf}$ in MO-CTL (5 ng) ( $\mathrm{n}=20)$ and MO-svep1 (5ng) $(\mathrm{n}=36)$ embryos treated with $1 \mathrm{X}$ tricaine $(0.014 \%)$ from 30 to $48 \mathrm{hpf}(\mathrm{N}=6)$. 

made available under aCC-BY-ND 4.0 International license.

(F) Bilateral quantifications of the percentage of lumenised segments in the DLAV at $48 \mathrm{hpf}$ in MO-CTL (5 ng) (n=20) and MO-svep1 (5ng) (n=36) embryos treated with $1 \mathrm{X}$ tricaine $(0.014 \%)$ from 30 to $48 \mathrm{hpf}(\mathrm{N}=6)$. 
Figure 2 - svep1 loss-of-function sensitises angiogenic remodelling to reduced blood flow.

(A)Bilateral quantifications of the percentage of gaps in the DLAV at $48 \mathrm{hpf}$ in MO-CTL (5 ng) and MO-svep1 (5ng) embryos treated with OX (n=14 MO-CTL, $\mathrm{n}=20$ MO-svep 1), 0.5X (0.007\%) (n=16 MO-CTL, $\mathrm{n}=24$ MO-svep1), $1 \mathrm{X}$ $(0.014 \%)(\mathrm{n}=22 \mathrm{MO}-\mathrm{CTL}, \mathrm{n}=27 \mathrm{MO}-\mathrm{svep} 1)$ or $2 \mathrm{X}(0.028 \%)(\mathrm{n}=21 \mathrm{MO}-\mathrm{CTL}$, $\mathrm{n}=27 \mathrm{MO}$-svep 1) tricaine from 30 to $48 \mathrm{hpf}(\mathrm{N}=3)$.

(B) Bilateral quantifications of the percentage of lumenised segments in the DLAV at $48 \mathrm{hpf}$ in MO-CTL (5 ng) and MO-svep1 (5ng) embryos treated with $0 \mathrm{X}$ $(\mathrm{n}=14 \mathrm{MO}-\mathrm{CTL}, \mathrm{n}=20 \mathrm{MO}-$ svep 1$), 0.5 \times(0.007 \%)(\mathrm{n}=16 \mathrm{MO}-\mathrm{CTL}, \mathrm{n}=24 \mathrm{MO}-$ svep1), $1 \times(0.014 \%)(\mathrm{n}=22 \mathrm{MO}-\mathrm{CTL}, \mathrm{n}=27 \mathrm{MO}$-svep1) or $2 \mathrm{X}(0.028 \%)(\mathrm{n}=21$ MO-CTL, $\mathrm{n}=27 \mathrm{MO}$-svep1) tricaine from 30 to $48 \mathrm{hpf}(\mathrm{N}=3)$.

(C) Bilateral quantifications of the percentage of gaps in the DLAV at $48 \mathrm{hpf}$ in MO-CTL (5 ng) (n=13), MO-gata1 (8ng) ( $\mathrm{n}=12)$, MO-svep1 (5ng) $(\mathrm{n}=16)$ and MO-gata1 (8ng)/MO-svep1 (5ng) $(\mathrm{n}=25)$ embryos $(\mathrm{N}=3)$.

(D)Bilateral quantifications of the percentage of lumenised segments in the DLAV at $48 \mathrm{hpf}$ in MO-CTL (5 ng) (n=13), MO-gata1 (8ng) $(\mathrm{n}=12), M O-$ svep1 (5ng) $(\mathrm{n}=16)$ and MO-gata1 (8ng)/MO-svep1 (5ng) $(\mathrm{n}=25)$ embryos $(\mathrm{N}=3)$. 
(E) Bilateral quantifications of the percentage of gaps in the DLAV at $48 \mathrm{hpf}$ in MO-CTL (5 ng) ( $\mathrm{n}=11)$, MO-tnnt2a (4ng) (n=12), MO-svep1 $(5 \mathrm{ng})(\mathrm{n}=12)$ and MO-tnnt2a (4ng)/MO-svep1 (5ng) ( $\mathrm{n}=21)$ embryos $(\mathrm{N}=3)$.

(F) Bilateral quantifications of the percentage of lumenised segments in the DLAV at $48 \mathrm{hpf}$ in MO-CTL (5 ng) (n=11), MO-tnnt2a (8ng) $(\mathrm{n}=12)$, MO-svep1 (5ng) $(\mathrm{n}=12)$ and MO-tnnt2a (4ng)/MO-svep1 (5ng) $(\mathrm{n}=21)$ embryos $(\mathrm{N}=3)$.

(G)Maximum intensity projection of representative DLAV in MO-CTL (5 ng) $(\mathrm{n}=11)$, MO-tnnt2a (8ng) $(\mathrm{n}=12)$, MO-svep1 (5ng) $(\mathrm{n}=12)$ and MO-tnnt2a (4ng)/MO-svep1 (5ng) ( $\mathrm{n=21}$ ) embryos at $48 \mathrm{hpf}$. Red asterisks indicate gaps. 
Figure 3 - svep1 loss-of-function leads to a defect in tip/stalk cell specification in primary angiogenic sprouts.

(A)Representative images of 48 hpf Tg(svep1:Gal4FF; UAS:eGFP); Tg(kdrl:mcherry-CAAX)y171 embryos with or without treatment with $1 \mathrm{X}$ $(0.014 \%)$ tricaine from 30 to $48 \mathrm{hpf}$.

(B) Quantification of average numbers of $\operatorname{Tg}$ (svep1:Gal4FF; UAS:eGFP) positive neurons in the neural tube area of $48 \mathrm{hpf}$ embryos with or without treatment with $1 X(0.014 \%)$ tricaine from 30 to $48 \mathrm{hpf}$. $(\mathrm{N}=3, \mathrm{n}=15$ controls, $\mathrm{n}=16$ treated).

(C) Maximum intensity projection of a representative TgBAC(apln:eGFP) $)^{\text {bns157, }}$ Tg(-0.8fIt1:RFP) ${ }^{\text {hu5333 }} \mathrm{MO}-\mathrm{CTL}$ (5ng) embryo. C' shows the unprocessed maximum intensity projection, C" shows the GFP signal volume-masked by the RFP signal, to limit detection to the endothelium and C"' shows the resulting endothelial GFP signal only.

(D) Maximum intensity projection of a representative TgBAC(apln:eGFP) $)^{\text {bns } 157 \text {, }}$ $\operatorname{Tg}(-0.8 f l t 1: R F P)^{h u 5333} M O-C T L(5 n g)$ embryo. D' shows the unprocessed maximum intensity projection, D" shows the GFP signal volume-masked by the RFP signal, to limit detection to the endothelium and D"' shows the resulting endothelial GFP signal only. 
(E) Maximum intensity projection of a MO-CTL (5ng) alSV at $48 \mathrm{hpf}$, highlighting the ventral and dorsal region used for further quantifications in (F) and (G).

(F) Quantification of the percentage of aISVs with apln:eGFP positive endothelial cells in the (1) dorsal and (2) ventral region in 48 hpf MO-CTL (5ng) $(n=10)$ and MO-svep1(5ng) $(\mathrm{n}=16)$ morphant embryos treated with $1 \mathrm{X}(0.014 \%)$ tricaine from 30 to $48 \mathrm{hpf}(\mathrm{N}=3)$.

(G)Quantification of the percentage of alSVs with more than one apln:eGFP positive endothelial cells in $48 \mathrm{hpf} M O-C T L(5 n g)(\mathrm{n}=10)$ and MO-svep1 (5ng) $(n=16)$ morphant embryos treated with $1 X(0.014 \%)$ tricaine from 30 to $48 \mathrm{hpf}$ $(\mathrm{N}=3)$.

(H) Representative image of $\mathrm{p}$-ERK and ERK levels in FAC sorted endothelial cells from MO-CTL (5ng) and MO-svep1 (5ng) morphants at 48hpf, treated with $1 \mathrm{X}(0.014 \%)$ tricaine from 30 to $48 \mathrm{hpf}(\mathrm{N}=4)$.

(I) Quantification of p-ERK in FAC sorted endothelial cells of MO-CTL (5ng) and MO-svep1 (5ng) morphants at 48hpf, treated with $1 \times(0.014 \%)$ tricaine from 30 to 48 hpf. Expression levels were normalised to pERK levels $(\mathrm{N}=4)$.

(J) Quantification of the percentage of aISVs with apln:eGFP positive endothelial cells in the (1) dorsal and (2) ventral region in $48 \mathrm{hpf}$ embryos treated with $1 \mathrm{X}$ $(0.014 \%)(n=22)$ or $2 X(0.028 \%)(n=24)$ tricaine from 30 to $48 \mathrm{hpf}(\mathrm{N}=3)$. 

made available under aCC-BY-ND 4.0 International license.

(K)Quantification of the percentage of alSVs with more than one apln:eGFP positive endothelial cells in $48 \mathrm{hpf}$ embryos treated with $1 \mathrm{X}(0.014 \%)(\mathrm{n}=22)$ or $2 \mathrm{X}(0.028 \%)(\mathrm{n}=24)$ tricaine from 30 to $48 \mathrm{hpf}(\mathrm{N}=3)$. 
Figure 4 - svep1 loss-of-function and knockdown are rescued by flt1 knockdown.

(A) Bilateral quantifications of the percentage of gaps in the DLAV at $48 \mathrm{hpf}$ in controls and mutant svep 1512-/- injected with MO-CTL (5ng) $(\mathrm{n}=45$ and $\mathrm{n}=27$ respectively) or MO-flt1 (1ng) ( $n=50$ and $n=12$ respectively), and treated with $1 \mathrm{X}$ tricaine $(0.014 \%)$ from 30 to $48 \mathrm{hpf}(\mathrm{N}=3)$.

(B) Bilateral quantifications of the percentage of gaps in the DLAV at $48 \mathrm{hpf}$ in MO-CTL (5 ng) (n=9), MO-flt1 (1ng) (n=7), MO-svep1 (5ng) $(\mathrm{n}=14)$ and MOflt1 (1ng)/MO-svep1 (5ng) $(\mathrm{n}=25)$ embryos $(\mathrm{N}=3)$.

(C) Bilateral quantifications of the percentage of lumenised segments in the DLAV at $48 \mathrm{hpf}$ in controls and mutant svep1512-/ injected with MO-CTL (5ng) ( $\mathrm{n}=45$ and $n=27$ respectively) or $M O-f l t 1$ ( $1 \mathrm{ng})$ ( $\mathrm{n}=50$ and $\mathrm{n}=12$ respectively), and treated with $1 \mathrm{X}$ tricaine $(0.014 \%)$ from 30 to $48 \mathrm{hpf}(\mathrm{N}=3)$.

(D) Bilateral quantifications of the percentage of lumenised segments in the DLAV at $48 \mathrm{hpf}$ in MO-CTL (5 ng) (n=9), MO-flt1 (1ng) $(\mathrm{n}=7)$, MO-svep1 (5ng) $(\mathrm{n}=14)$ and MO-flt1 (1ng)/MO-svep1 (5ng) $(\mathrm{n}=25)$ embryos, treated with $1 \mathrm{X}$ tricaine (0.014\%) from 30 to $48 \mathrm{hpf}(\mathrm{N}=3)$.

(E) Bilateral quantifications of the percentage of aISV loops at $48 \mathrm{hpf}$ in $M O-C T L$ (5 ng) $(\mathrm{n}=11)$, MO-flt1 (1ng) $(\mathrm{n}=14)$, MO-svep1 (5ng) $(\mathrm{n}=11)$ and MO-flt1 
$(1 \mathrm{ng}) / \mathrm{MO}$-svep1 $(5 \mathrm{ng})(\mathrm{n}=20)$ embryos treated with $1 \mathrm{X}$ tricaine $(0.014 \%)$ from 30 to 48 hpf $(\mathrm{N}=3)$.

(F) Representative image of an arterial alSV loop in MO-svep1(5ng)/MO-flt1(1ng)

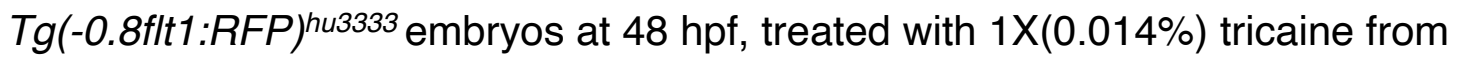
30 to 48 hpf. Tg(-0.8flt1:RFP)hu333

(G)Quantification of number of nucleus per loop area (see figure 4F) at $48 \mathrm{hpf}$ in MO-svep1(5ng)/MO-flt1(1ng) embryos at $48 \mathrm{hpf}$, treated with $1 \mathrm{X}(0.014 \%)$ tricaine from 30 to $48 \mathrm{hpf}(\mathrm{n}=34$ loops counted. $2,19,9$ and 4 loops had 1, 2, 3 or 4 nucleus per loop area, respectively). 20/34 loops were lumenised) ( $N=3)$

(H) Representative image of an alSV to alSV connection in the region of the horizontal myoseptum at $48 \mathrm{hpf}$ in in MO-svep1(5ng)/MO-flt1(1ng) $\mathrm{Tg}(-$

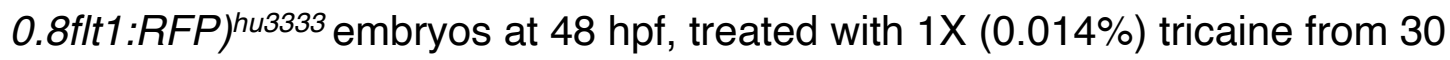
to 48 hpf. ( $\mathrm{n}=20$ fish, 26 connections visible out of 280 somites, $8 / 26$ connections were lumenised, $\mathrm{N}=3$ ).

(I) Maximum intensity projection of a representative TgBAC(apln:eGFP) $)^{\text {bns } 157 \text {, }}$

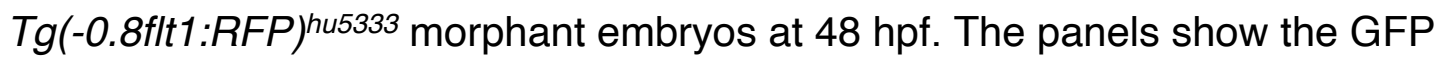
signal volume-masked by the RFP signal, to limit detection to the endothelium in MO-CTL (5ng), MO-flt1 (1ng), MO-svep1 (5ng) and MO-svep1 (5ng) /MOflt1 (1ng) embryos treated with $1 \mathrm{X}(0.014 \%)$ tricaine from 30 to $48 \mathrm{hpf}$. 
(J) Maximum intensity projection of a MO-svep1 (5ng)/MO-flt1 (1ng) alSV at 48 hpf, highlighting the ventral and dorsal region used for further quantifications in (K).

(K)Quantification of the percentage of alSVs with apln:eGFP positive endothelial cells in the (1) dorsal and (2) ventral region in $48 \mathrm{hpf} M O-C T L(5 n g)(n=12)$, MO-flt1 (1ng) ( $\mathrm{n}=14)$, MO-svep1 $(5 \mathrm{ng})(\mathrm{n}=12)$ and MO-svep1(5ng)/MO-flt1 (1ng) $(n=20)$ morphant embryos treated with $1 X(0.014 \%)$ tricaine from 30 to $48 \mathrm{hpf}(\mathrm{N}=3)$.

(L) Quantification of the percentage of alSVs with more than one apln:eGFP positive endothelial cells in $48 \mathrm{hpf} M O-C T L(5 n g)(n=12), M O-f l t 1(1 n g)(n=14)$, MO-svep1 (5ng)( $\mathrm{n}=12)$ and MO-svep1 (5ng)/MO-flt1 (1ng) $(\mathrm{n}=20)$ morphant embryos treated with $1 \mathrm{X}(0.014 \%)$ tricaine from 30 to $48 \mathrm{hpf}(\mathrm{N}=3)$. 
Figure 5 - Vegfa/Vegfr signalling is necessary for ISV lumenisation maintenance and DLAV formation

(A) Maximum intensity projections at $48 \mathrm{hpf}$ of the trunk of MO-CTL (5ng) and MO-svep1 (5ng), Tg(fli1a:eGFP)y7 embryos, treated with 1X $(0.014 \%)$ tricaine, with or without 50ng ZM32881.

(B) Bilateral quantifications of the percentage of gaps in the DLAV at $48 \mathrm{hpf}$ in MO-CTL (5 ng) (n=29 (0nM ZM32881), n=28 (50nM ZM32881)) MO-svep1 (5ng) (n=29 (0 nM ZM32881), n=26 (50nM ZM32881)) embryos treated with $1 \mathrm{X}(0.014 \%)$ tricaine and 0 or $50 \mathrm{nM}$ ZM32881 from 30 to $48 \mathrm{hpf},(\mathrm{N}=3)$.

(C) Bilateral quantifications of the percentage of lumenised segments in the DLAV at $48 \mathrm{hpf}$ in MO-CTL (5 ng) ( $\mathrm{n}=29$ (0nM ZM32881), $\mathrm{n}=28$ (50nM ZM32881)) MO-svep1 (5ng) (n=29 (0 nM ZM32881), n=26 (50nM ZM32881)) embryos treated with $1 \mathrm{X}(0.014 \%)$ tricaine and 0 or $50 \mathrm{nM}$ ZM32881 from 30 to $48 \mathrm{hpf}$ $(\mathrm{N}=3)$.

(D) Representative images of missing ISVs, fully lumenised ISV and not fully lumenised ISV at $48 \mathrm{hpf}$. Quantifications of these phenotypes are presented in (E) and (F).

(E) Bilateral quantifications of the percentage of missing ISVs in the trunk of 48 hpf MO-CTL (5 ng) (n=29 (OnM ZM32881), n=28 (50nM ZM32881)) MO- 
svep1 (5ng) ( $\mathrm{n}=29$ (0 nM ZM32881), $\mathrm{n}=26$ (50nM ZM32881)) embryos treated

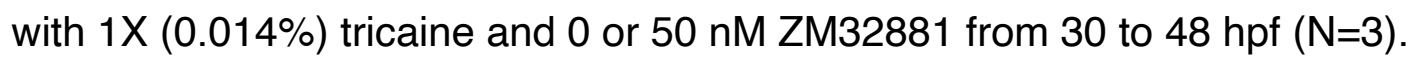

(F) Bilateral quantifications of the percentage of ISVs lumenised dorsally to ventrally in the trunk of 48 hpf MO-CTL (5 ng) (n=29 (OnM ZM32881), n=28 (50nM ZM32881)) MO-svep1 (5ng) (n=29 (0 nM ZM32881), $\mathrm{n}=26$ (50nM ZM32881)) embryos treated with $1 \mathrm{X}(0.014 \%)$ tricaine and 0 or $50 \mathrm{nM}$ ZM32881 from 30 to $48 \mathrm{hpf}(\mathrm{N}=3)$.

(G)Bilateral quantifications of the percentage of gaps in the DLAV at $48 \mathrm{hpf}$ in embryos treated with $2 X(0.028 \%)$ tricaine and $0(n=22)$ or $50 \mathrm{nM}(n=21)$ ZM32881 from 30 to $48 \mathrm{hpf},(\mathrm{N}=3)$.

(H)Bilateral quantifications of the percentage of lumenised segments in the DLAV at $48 \mathrm{hpf}$ in embryos treated with $2 \mathrm{X}(0.028 \%)$ tricaine and $0(\mathrm{n}=22)$ or $50 \mathrm{nM}$ $(\mathrm{n}=21) \mathrm{ZM} 32881$ from 30 to $48 \mathrm{hpf},(\mathrm{N}=3)$

(I) Bilateral quantifications of the percentage of missing ISVs in the trunk of 48 hpf in embryos treated with $2 X(0.028 \%)$ tricaine and $50 \mathrm{nM}(\mathrm{n}=21) \mathrm{ZM} 32881$ from 30 to $48 \mathrm{hpf},(\mathrm{N}=3)$.

(J) Bilateral quantifications of the percentage of ISVs lumenised dorsally to ventrally in the trunk of $48 \mathrm{hpf}$ in embryos treated with $2 \mathrm{X}(0.028 \%)$ tricaine and $50 \mathrm{nM}(\mathrm{n}=21) \mathrm{ZM} 32881$ from 30 to $48 \mathrm{hpf},(\mathrm{N}=3)$. 
bioRxiv preprint doi: https://doi.org/10.1101/2021.03.13.435246; this version posted March 19, 2021. The copyright holder for this preprint (which was not certified by peer review) is the author/funder, who has granted bioRxiv a license to display the preprint in perpetuity. It is made available under aCC-BY-ND 4.0 International license. 
Firu 2 bioRxiv preprint doi: https://doi.org/10.1101/2021.03.13.435246; this version posted March 19, 2021. The copyright holder for this preprint

(which was not certified by peer review) is the author/funder, who has granted bioRxiv a license to display the preprint in perpetuity. It is

A
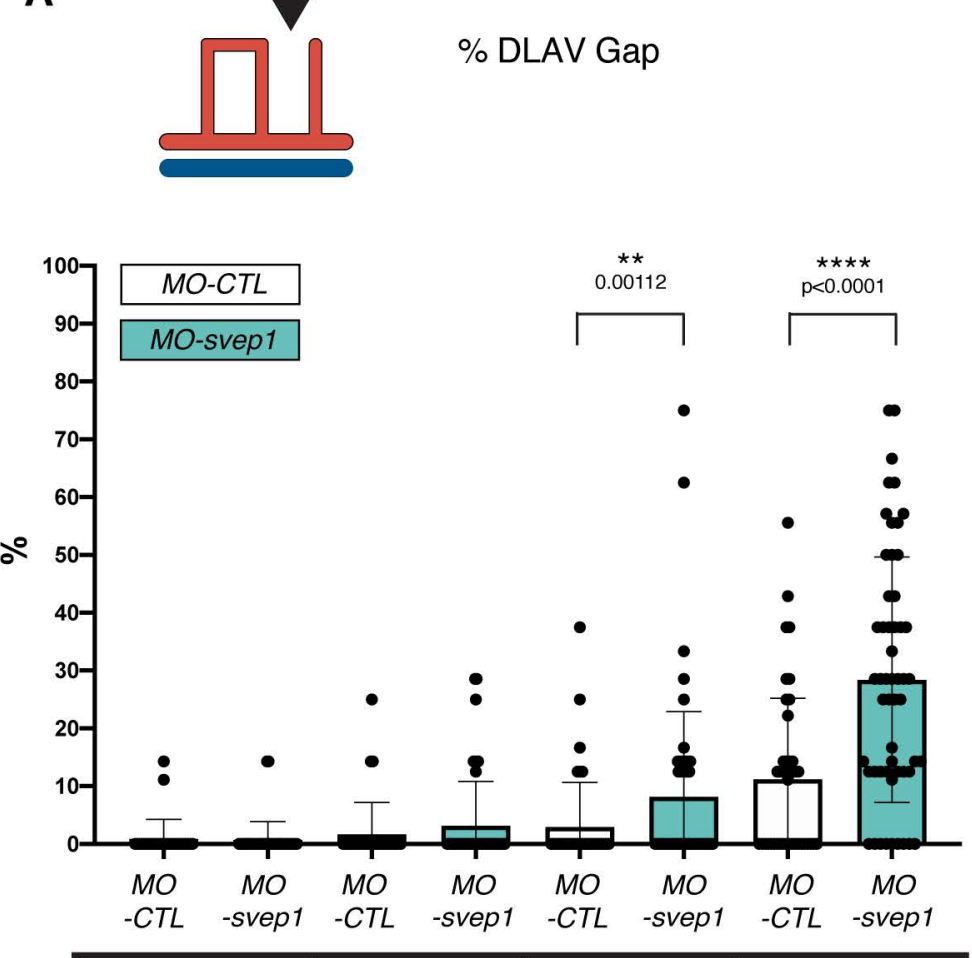

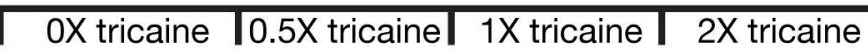

slower blood flow (30-48 hpf treatment with tricaine)
BD 4.0 International license.

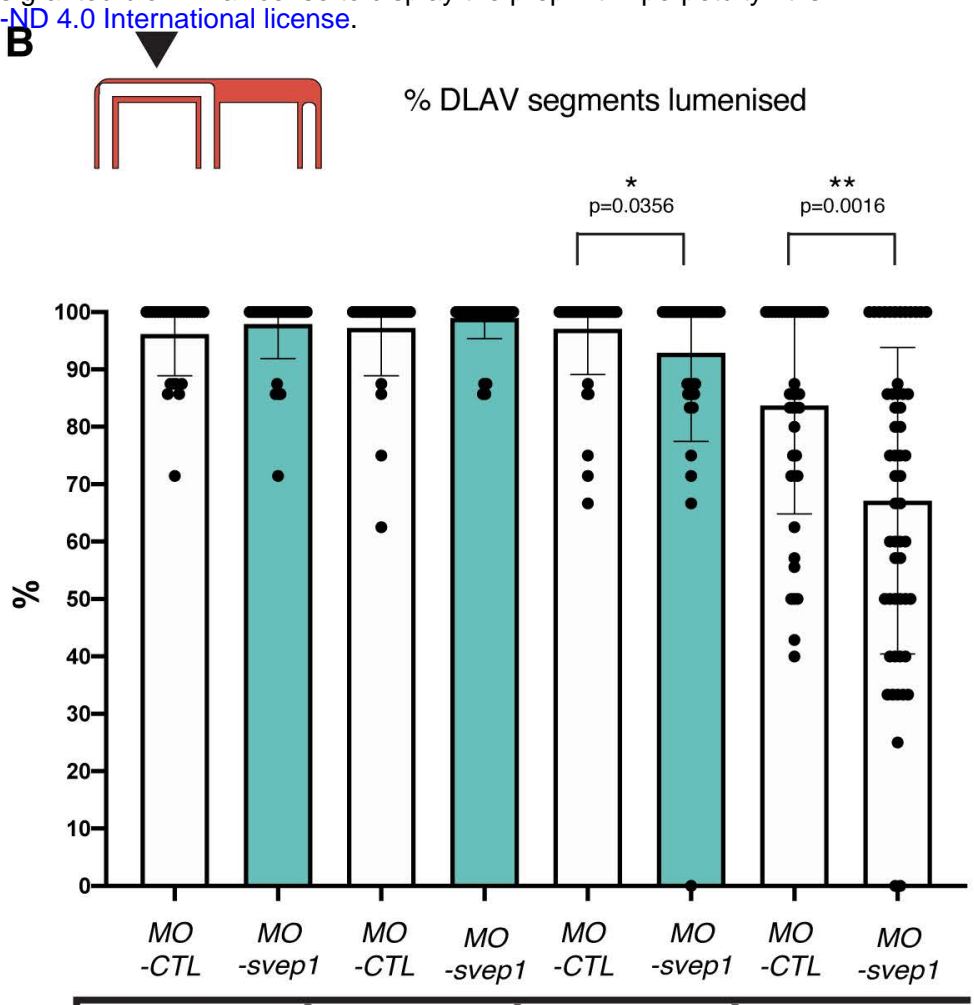

0X tricaine $10.5 \mathrm{X}$ tricaine $\mathrm{IX}$ tricaine $\mathrm{I} 2 \mathrm{X}$ tricaine

slower blood flow (30-48 hpt treatment with tricaine)

\% DLAV

G MO-CTL

MO-tnnt2a

MO-svep1

D

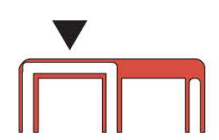

segments

lumenised

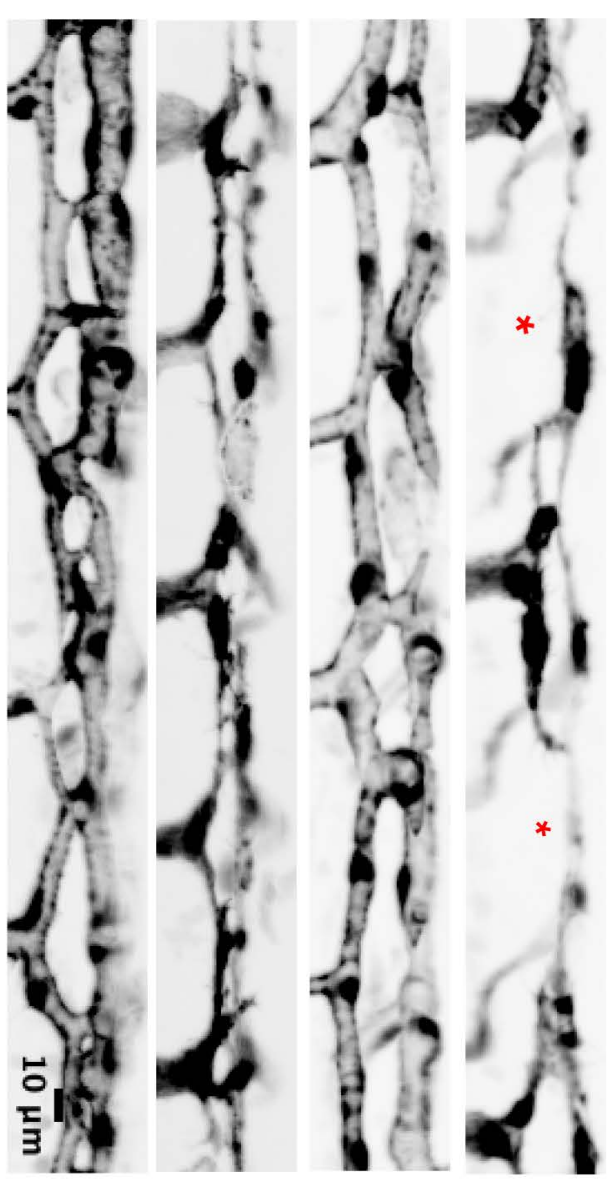

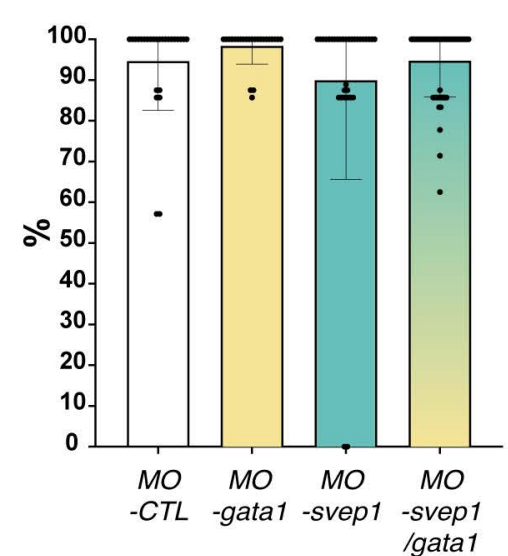

F

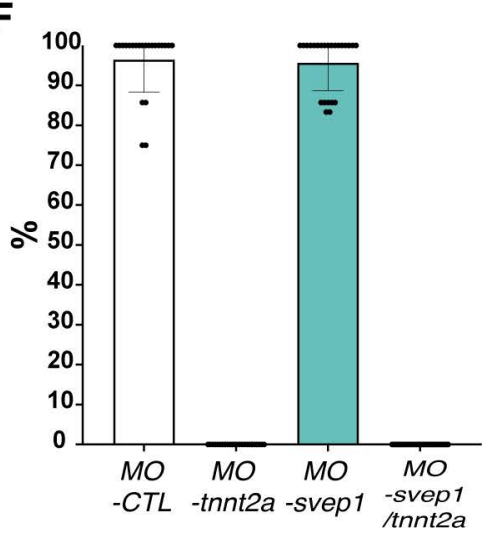

\section{MO-svep1}

MO-tnnt2a
E

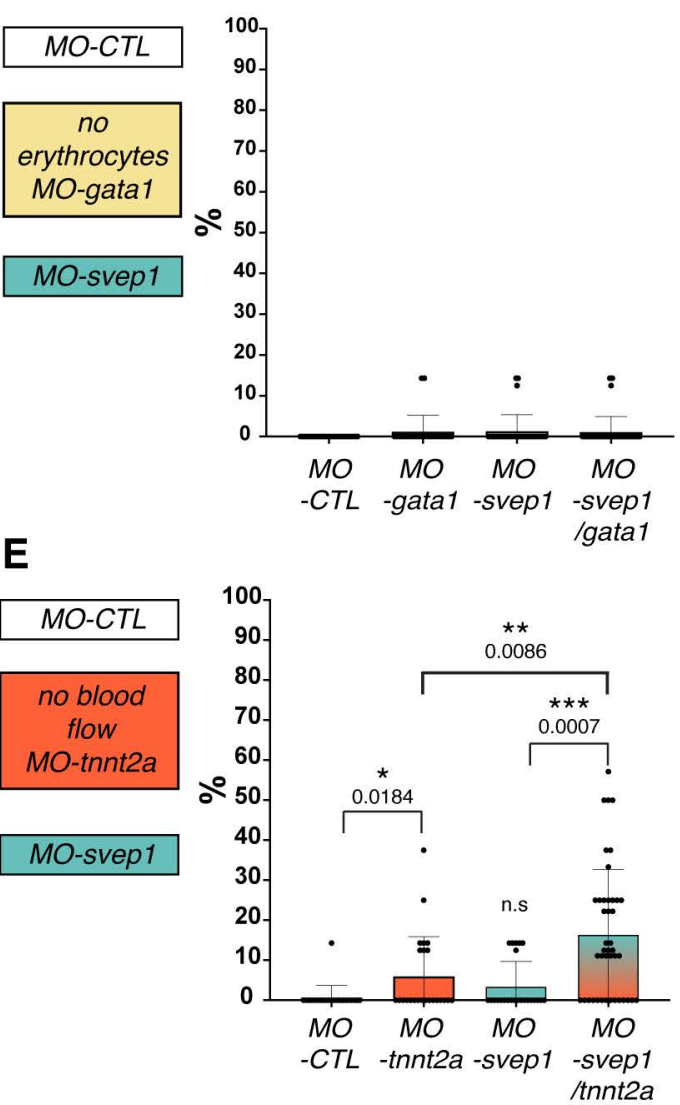


Figure 3 bioRxiv preprint doi: https://doi.org/10.1101/2021.03.13.435246; this version posted March 19, 2021. The copyright holder for this. preprint

A

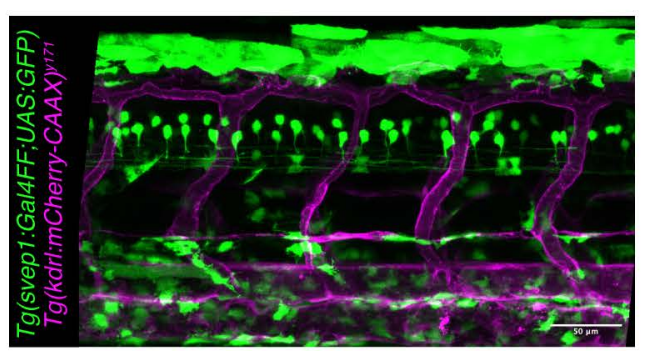

made available under aCC-BY-ND 4.0 International license.

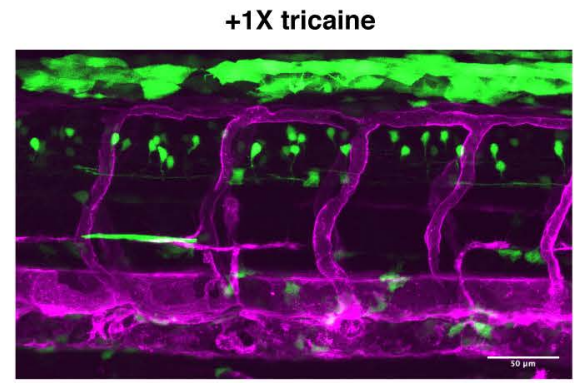

$+1 \mathrm{X}$ tricaine (30 to $48 \mathrm{hpf}$ )
B

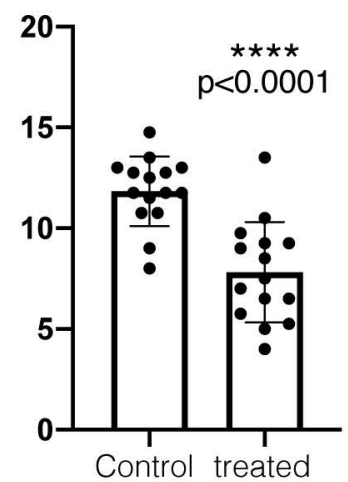

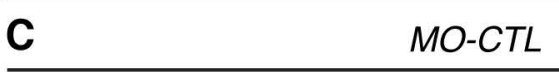
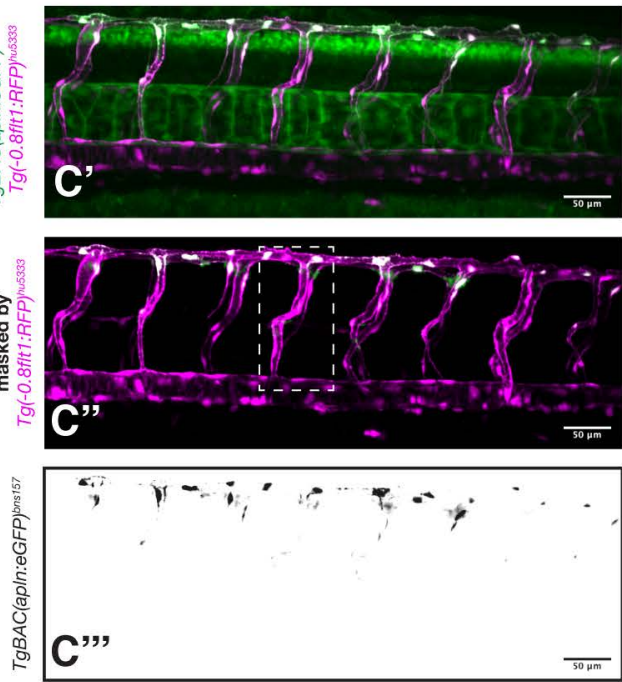

E

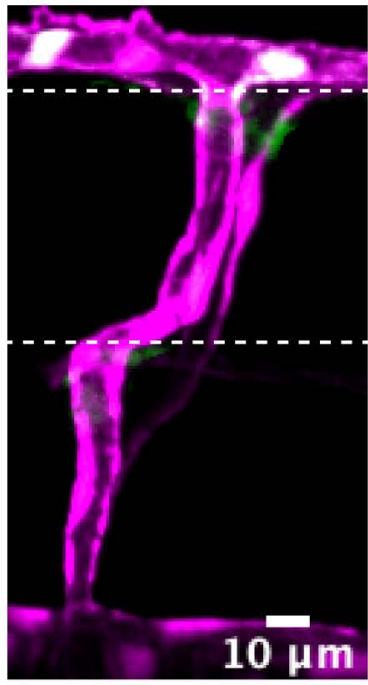

$M O-C T L$

H

Ventral

region

(2)

(1)

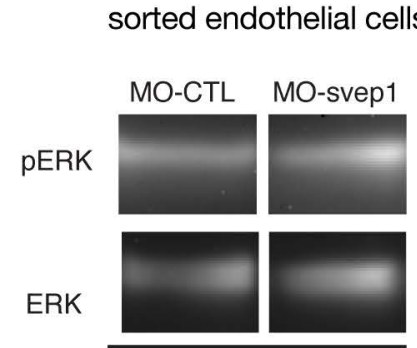

$+1 \mathrm{X}$ Tricaine 30-48 hpf

p-ERK/ERK

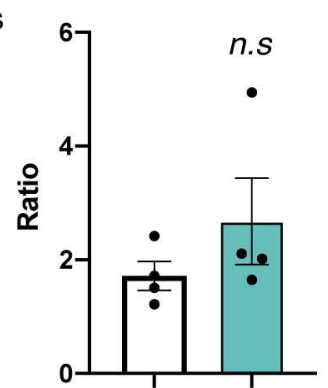

D
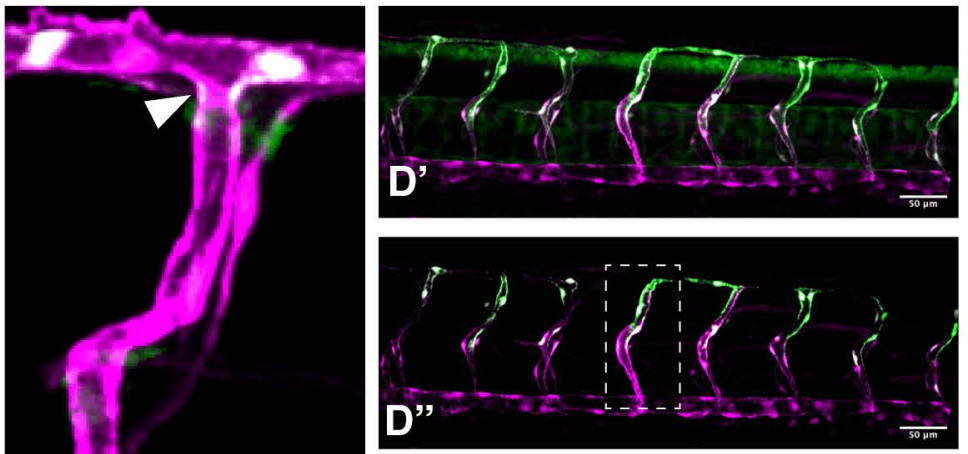

$10 \mu \mathrm{m}$
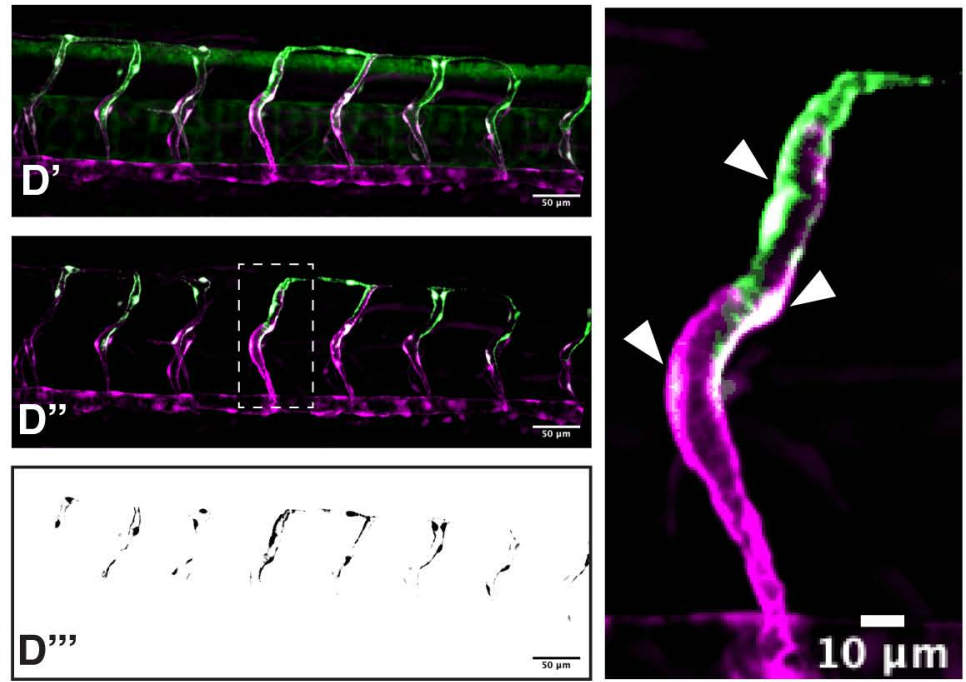

$\mathbf{F}$

- $\quad \%$ alSVS with apln:eGFP positive EC

G

$\%$ alSV with $>1$ ap/n:eGFP positive EC

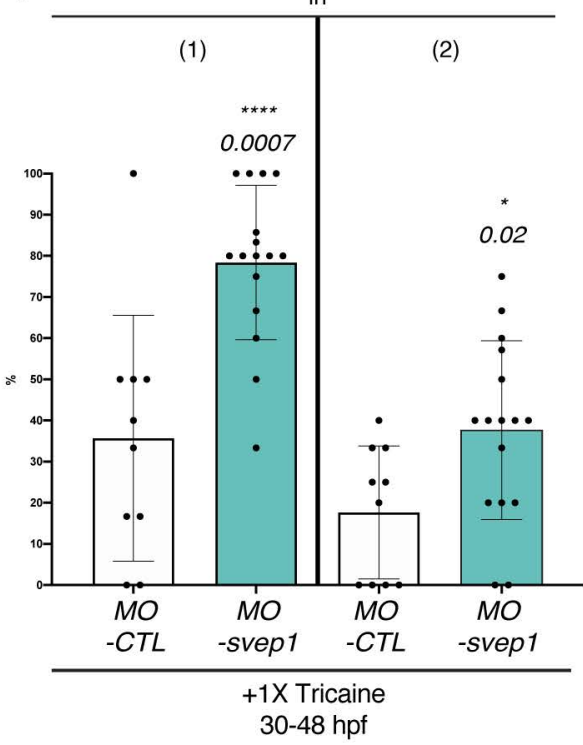

J

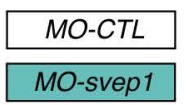

$<0.0001$

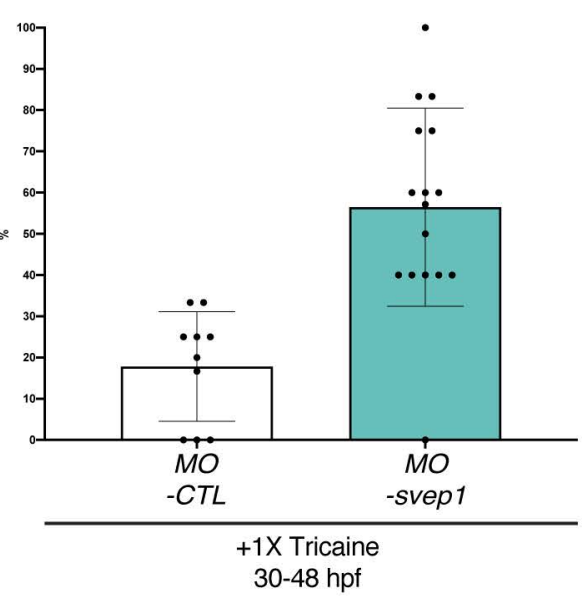

$\mathbf{K}$

$\%$ alSV with $>1$ apln:eGFP positive EC
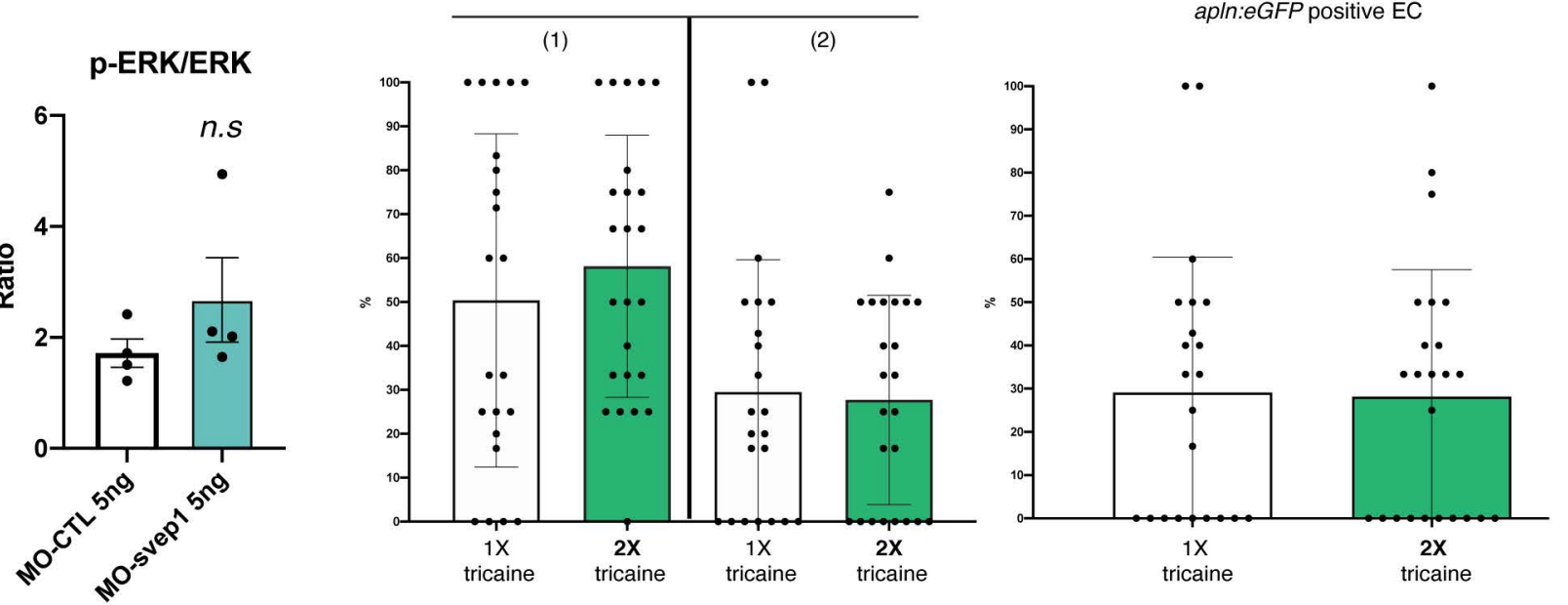
bioRxiv preprint doi: https://doi.org/10 $101 / 2021.03 .13 .435246$; this version posted March $\$ \nabla, 2021$. The copyright holder for this preprint Figure 4 (which was not certified by peer review) is the author/funder, who has granted bioRxiv aticense to display the preprintinsperpeteity lt is

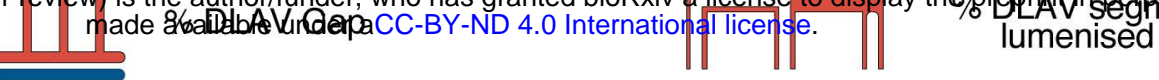

A

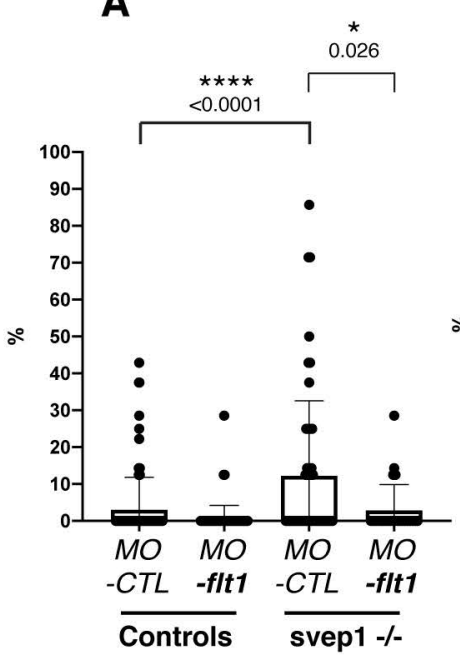

E $\quad \%$ arterial loops $\frac{\substack{\star \star \star \star * \star \\<0.001}}{\Delta 0.0001}$

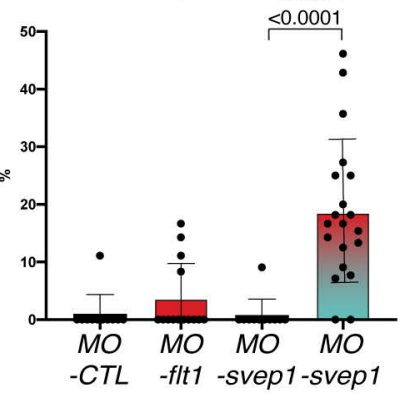

B

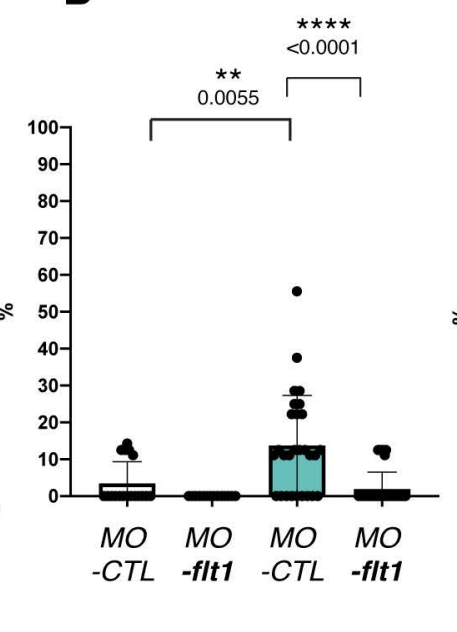

C

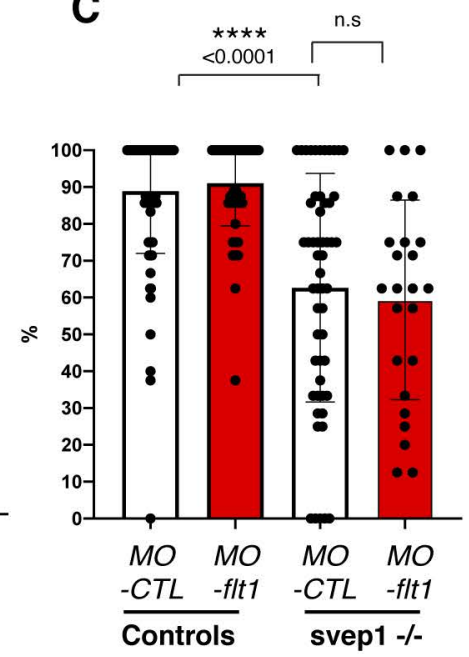

G (1) Number of nucleus per loop area<smiles>CC(F)=[W]</smiles>

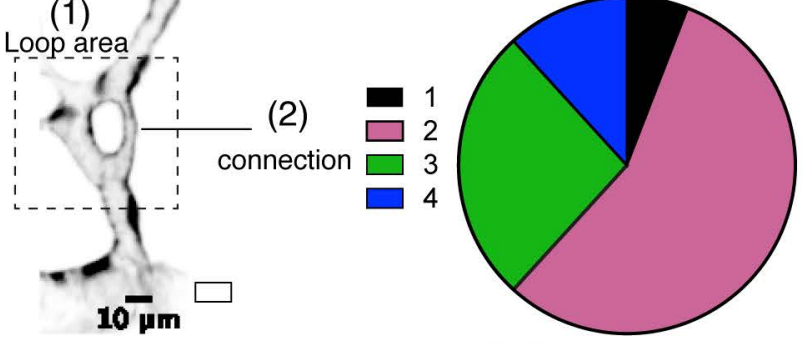

(20/34 loops lumenised)

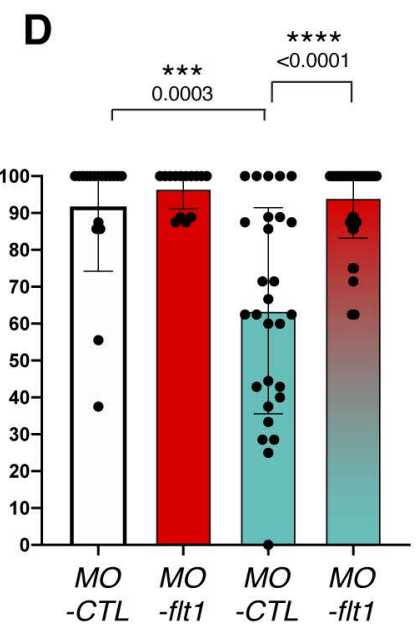

H

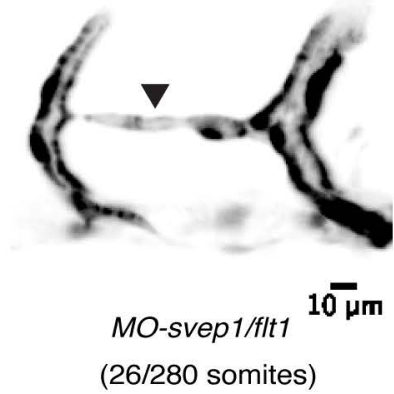

$+1 \mathrm{X}$ tricaine (30 to $48 \mathrm{hpf})$
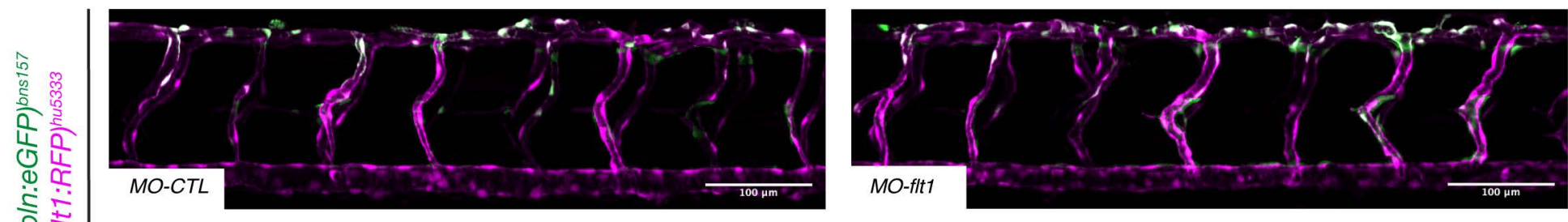

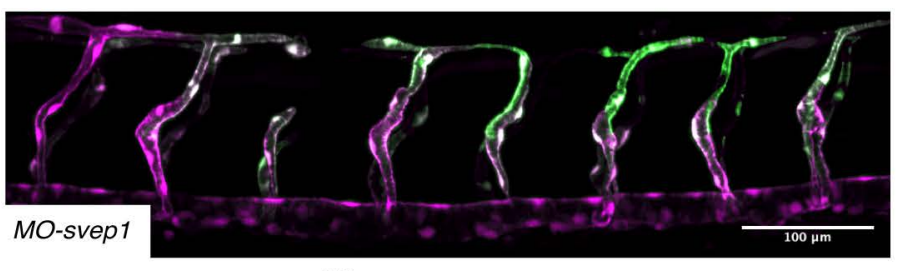

K

$\%$ alSVS with apln:eGFP positive EC

\section{J}

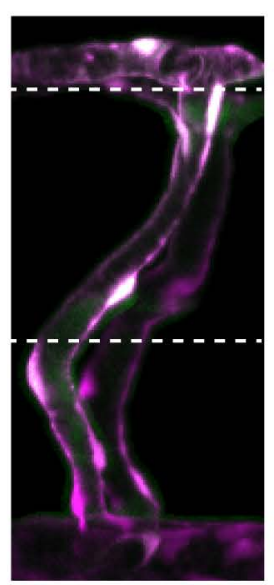

MO-svep1/MO-flt1
(1) *

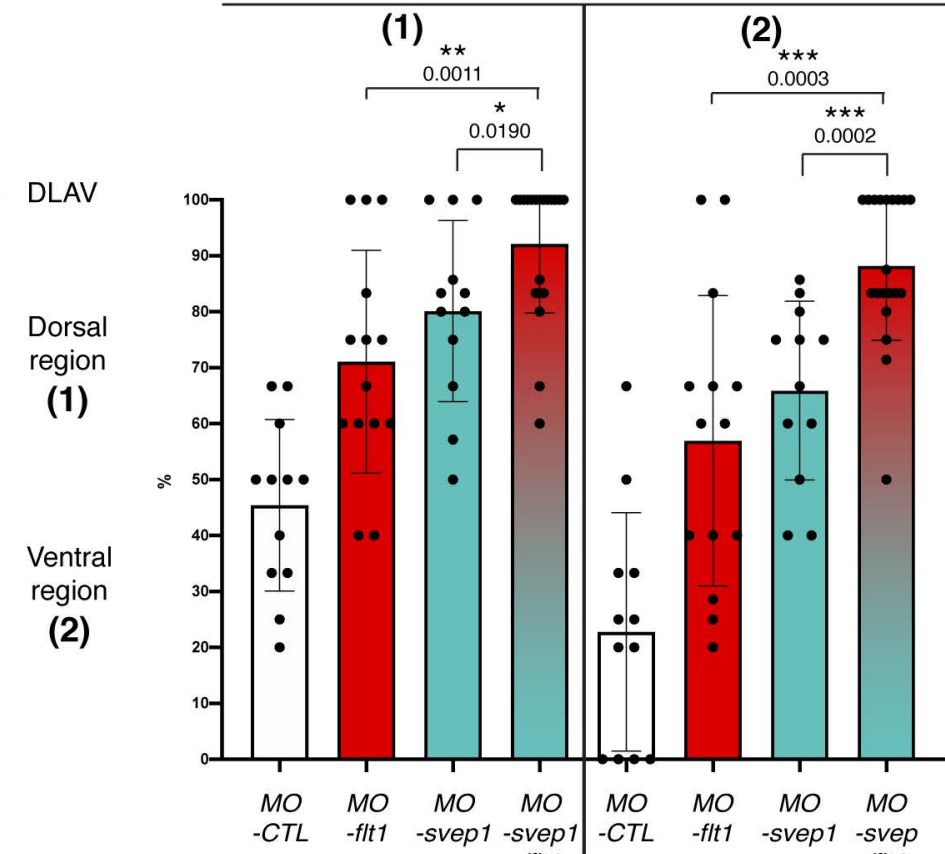

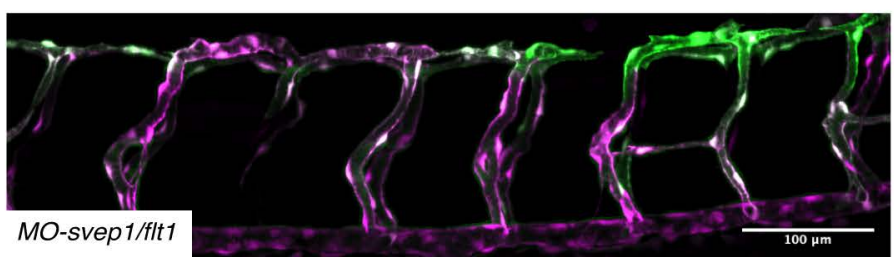

L

$\%$ alSV with $>1$ apln:eGFP positive EC

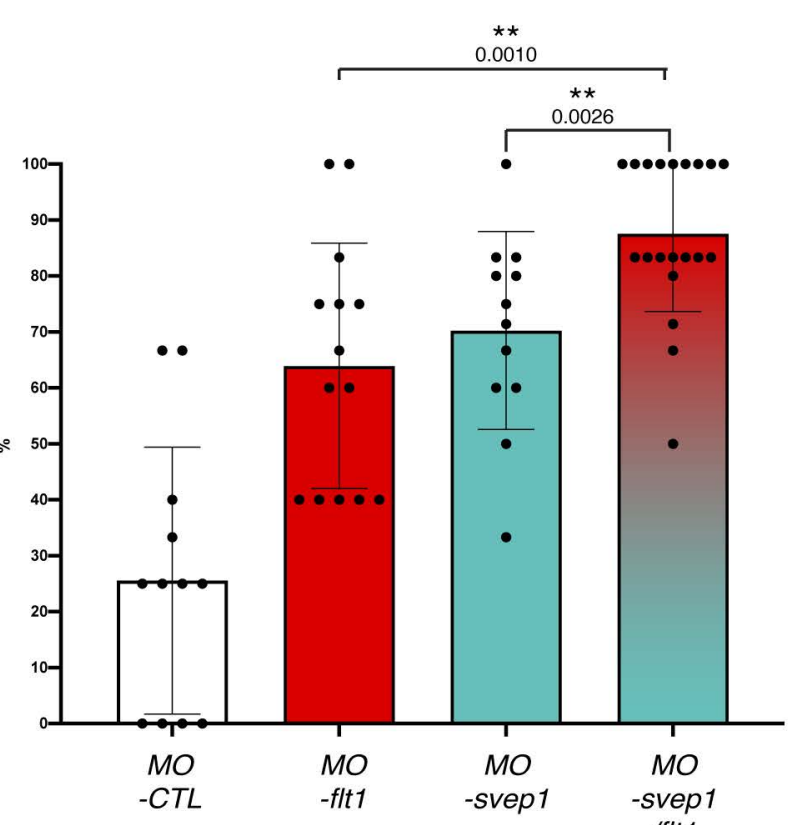


